\title{
Bézier tilings of the sphere and their applications in benchmarking multipatch isogeometric methods
}

\author{
Sander Dedoncker ${ }^{\mathrm{a}, 1, *}$, Laurens Coox $^{\mathrm{a}, 1}$, Florian Maurin ${ }^{\mathrm{a}, 1}$, Francesco Greco ${ }^{\mathrm{a}, 1}$, \\ Wim Desmet ${ }^{\mathrm{a}, 1}$ \\ ${ }^{a}$ KU Leuven, Department of Mechanical Engineering, Celestijnenlaan 300 box 2420, B-3001 \\ Leuven, Belgium
}

\begin{abstract}
In this work, we explore new parametric models of a sphere with rational Bézier patches. The geometry is represented as a spherical tessellation with various topologies. The tiles are degeneracy-free rational Bézier surfaces, obtained using a general composite mapping method. Five novel, topologically distinct tilings are described, four of which have conforming parameterizations. The mesh of the fifth tiling is nonconforming despite having equal interface knot vectors (intrinsic nonconformity). We apply these novel geometries in three case studies that are proposed as benchmark problems for $C^{0}$ - and $C^{1}$-multipatch IGA methods.
\end{abstract}

Keywords: Bézier geometry, Spherical tiles, Isogeometric analysis, Patch coupling, Benchmark

\section{Introduction}

As product requirements are becoming more stringent and design iterations more frequent, the reliance on digital tools is ever increasing. Both in computer aided design (CAD) and in computer aided engineering (CAE), these tools are already indispensable. However, because CAD and CAE originated separately, both fields employ different methods and technologies. This has created a bottleneck in the design process since translation of the models is generally a highly time-consuming task. In their seminal paper [1], Hughes et al. proposed a new paradigm that aims to overcome this conflict. In isogeometric analysis (IGA), the fundamental distinction between design and engineering models is eliminated by using the same mathematical framework to express both. Since its introduction,

\footnotetext{
${ }^{*}$ Corresponding author

Email address: sander.dedoncker@kuleuven.be (Sander Dedoncker)

${ }^{1}$ Member of Flanders Make
} 
IGA has been subjected to extensive investigation [2] and a monograph has been published [3]. The literature indicates that the fusion between CAD and CAE is a promising concept but shows there are still hurdles to overcome.

Since their inception and popularization in the second half of the twentieth century, Bézier curves and surfaces have evolved to become the standard technology in the CAD industry. Undoubtedly, one of their biggest merits is the very intuitive freeform modelling they allow. Moreover, the generalization to rational Bézier patches and B-splines (non-uniform rational B-splines or NURBS) has enabled designers to represent common geometries such as quadrics exactly [4]. The problem of representing these surfaces becomes a problem of finding the right control points and weights. Almost thirty years ago, Cobb [5] obtained a degeneracy-free Bézier tessellation of the simplest quadric surface: the sphere. In his work, however, not all possible regular topologies are explored. The primary aim of the present paper is to describe additional Bézier tessellations of the sphere using Cobb's methodology, and to provide an algorithm for generating spherical tiles from four arbitrary vertex points.

The tensor product structure that is inherent to NURBS patches limits singlepatch geometries to relatively simple shapes. In a realistic design context, the geometries are usually far too complex to be represented by a single unmodified NURBS patch. To overcome this problem, patches may be trimmed and multiple patches may be stitched together. A CAD model thus typically consists of a multipatch (and possibly trimmed) NURBS representation, made mostly without analysis in mind. Some authors therefore propose to generalize or replace NURBS technology used in CAD in order to make isogeometric analysis more feasible, for instance, using T-splines [6, 7], subdivision surfaces [8, 9] and B++-splines [10]. However, reliance on new and nonstandard technology also heightens the threshold for the industrial acceptance of IGA. The problem can be alleviated by using separate discretizations for the geometry and for the fields [11, 12], but this approach still relies on shape functions other than NURBS to represent fields in a multipatch geometry. Developing techniques to couple NURBS patches thus remains highly important for isogeometric analysis.

A large body of research has been devoted to the patch coupling problem and different techniques have been developed - see [13, 14] for a detailed overview. Some methods, such as penalty methods [15], Lagrange multiplier methods [16], Nitsche's method [17, 18] and mortar methods [19 21], enforce weakly the Dirichlet interface constraint with various approaches. The VUKIMS method proposed by Coox et al. [13] also enforces interface constraints in a weak sense, but does so by explicitly making use of NURBS refinement properties. The particularly complicated problem of $C^{1}$-coupling is discussed in [22 27]. Depending on the technique used, extra variables may be introduced, expensive additional calculations may 
be needed and/or the conditioning of the final system may be affected. No (de facto) standard method has been adopted, likely because of the difficult tradeoffs involved. There seem to be few general benchmark cases for comparing the different coupling methods, despite the abundant research. The secondary goal of the present work is to address this lack of benchmark problems in multipatch-IGA.

The remainder of the paper is structured as follows: in Section 2, the composite mapping method for generating spherical tiles is applied to a general quadrangular tile. This method is used in Section 3 to generate novel and degeneracy-free spherical tilings based on different topologies, in particular those derived from the Platonic and Catalan solids. In Section 4, both complete and partial tilings are applied to three different problems which may serve as benchmark cases for multipatch isogeometric methods. Section 5 concludes the text.

\section{The composite mapping method}

Following the development by Cobb [5], a composite mapping technique for generating rational Bézier representations of spherical tiles is elaborated. This method is built around the stereographic projection, which is a well-known mapping in geometry [28]. After defining the projection in Euclidean space, the transition to the projective space is made. This is useful because rational Bézier patches are more elegantly described as polynomial Bézier patches in the projective space [4]. Finally, a general method for making quadrangular sphere tiles is outlined. Some particular points of attention are addressed along the derivation.

\subsection{Stereographic projection in Euclidean space}

The stereographic projection is defined using the geometric objects depicted in Fig. 1. Let $\Sigma$ be the unit sphere in $\mathbb{R}^{3}$

$$
\Sigma=\left\{(x, y, z) \in \mathbb{R}^{3} \mid x^{2}+y^{2}+z^{2}=1\right\},
$$

and let $\Pi$ be the plane tangent to the south pole of $\Sigma$

$$
\Pi=\left\{(x, y, z) \in \mathbb{R}^{3} \mid z=-1\right\} .
$$

The point $P=(u, v,-1)$ in the tangent plane and the north pole $N=(0,0,1)$ define a line $\Lambda_{r}$

$$
\Lambda_{r}=\left\{(x, y, z) \in \mathbb{R}^{3} \mid x=t u, y=t v, z=1-2 t, \forall t \in \mathbb{R}\right\},
$$

which intersects the sphere in two points (one of which is the north pole)

$$
\Lambda_{r} \cap \Sigma=\left\{(0,0,1),\left(\frac{4 u}{u^{2}+v^{2}+4}, \frac{4 v}{u^{2}+v^{2}+4}, \frac{u^{2}+v^{2}-4}{u^{2}+v^{2}+4}\right)\right\} .
$$




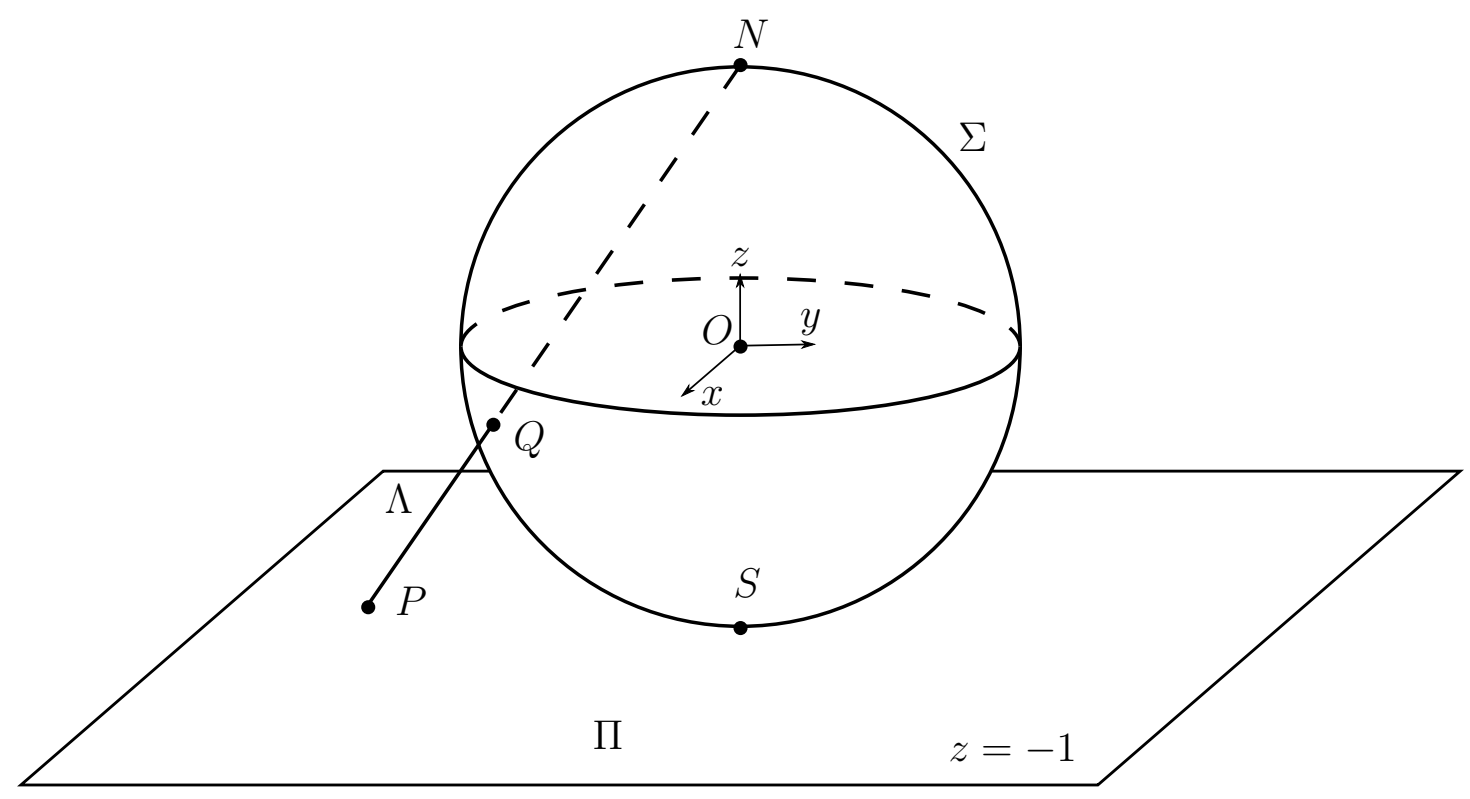

Figure 1: Geometric representation of the stereographic projection

Hence the intersection point on the sphere can be given as a function of the location of $P$. This leads to the definition of the map $r$

$$
r: \mathbb{R}^{2} \rightarrow \mathbb{R}^{3},(u, v) \rightarrow\left(\frac{4 u}{u^{2}+v^{2}+4}, \frac{4 v}{u^{2}+v^{2}+4}, \frac{u^{2}+v^{2}-4}{u^{2}+v^{2}+4}\right),
$$

which is called the inverse stereographic projection. Conversely, a point $Q=$ $\left(x_{Q}, y_{Q}, z_{Q}\right) \in \Sigma$ together with $N$ determines a line $\Lambda_{s}$

$$
\Lambda_{s}=\left\{(x, y, z) \in \mathbb{R}^{3} \mid x=t x_{Q}, y=t y_{Q}, z=1+\left(z_{Q}-1\right) t, \forall t \in \mathbb{R}\right\},
$$

such that

$$
\Lambda_{s} \cap \Pi=\left\{\left(\frac{2 x_{Q}}{1-z_{Q}}, \frac{2 y_{Q}}{1-z_{Q}},-1\right)\right\} .
$$

A second mapping $s$ (the stereographic projection),

$$
s: \mathbb{R}^{3} \rightarrow \mathbb{R}^{2},(x, y, z) \rightarrow\left(\frac{2 x}{1-z}, \frac{2 y}{1-z}\right),
$$

is then the inverse of $r$. Note that both $r$ and $s$ are rational maps.

The stereographic projection has some useful properties. First of all, it can be shown (see Section 2.4) that this function maps great circle arcs (geodesics) to circle arcs or line segments; this property is shown in Fig. 2. It follows that any spherical polygon - a shape bounded by a finite number of great arcs - will be 


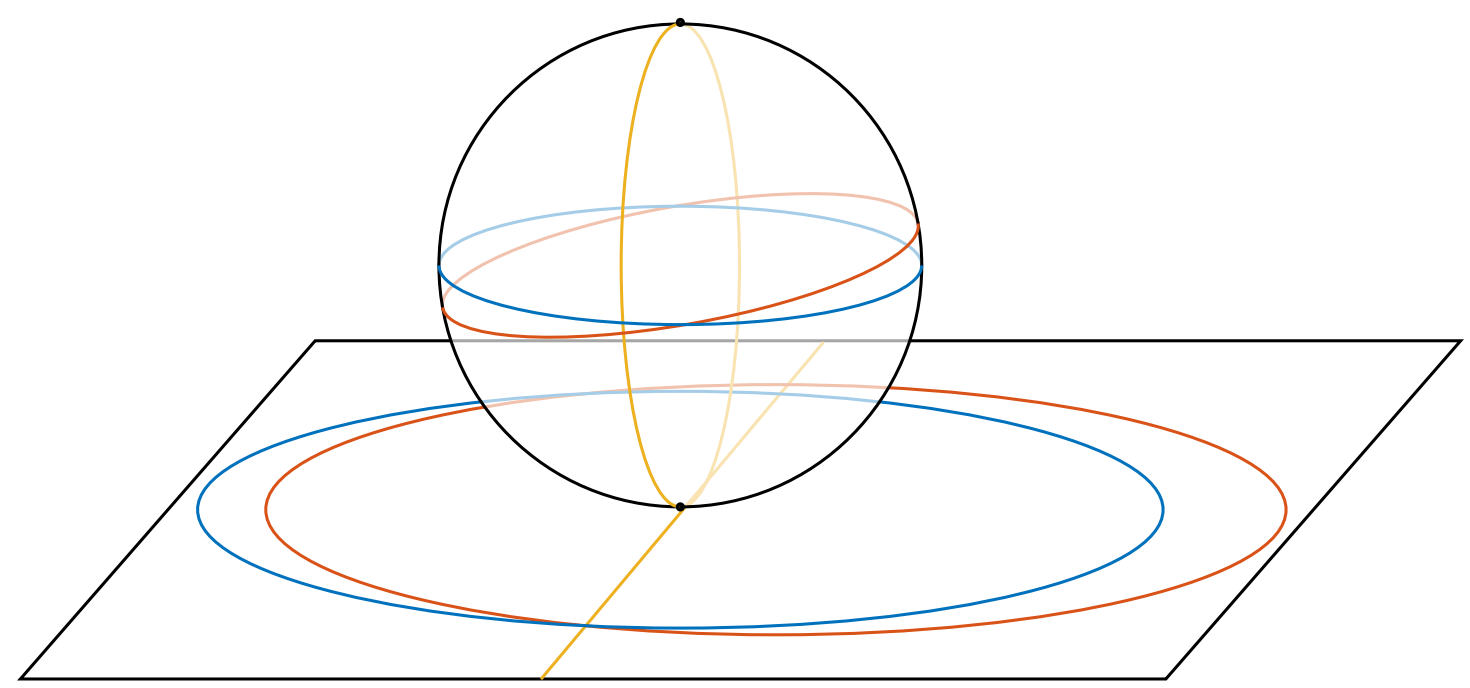

Figure 2: The stereographic projection maps great circles on the unit sphere to circles or lines in the projection plane.

projected onto a region bounded by circle arcs. Moreover, the projected arcs will always curve towards the origin. In other words, the origin is always in the circle constructed by completing the arcs. Secondly, the map is conformal: the angles between curves are preserved in the transformation [5]. In conclusion, it can be stated that a convex polygon containing the south pole is projected onto a convex region containing the origin, since i) sphere polygons are geodesically convex if and only if the interior angles are less than $180^{\circ}$ and ii) the bounding arcs curve towards the origin.

\subsection{Stereographic projection in projective space}

The real projective $n$-space $\mathbb{R P}^{n}$ is formally defined as the quotient space of $\mathbb{R}_{0}^{n+1}$ with equivalence relation $X \sim \lambda X, \lambda \neq 0$ [28]. In other words, a 'point' in $\mathbb{R} \mathbb{P}^{n}$ corresponds to a line through the origin in $\mathbb{R}^{n+1}$. Elements of $\mathbb{R} \mathbb{P}^{n}$ can be represented by a set of homogeneous coordinates $\left[x_{1}, x_{2}, \ldots, x_{n+1}\right]$, not all $x_{i}=0$ such that

$$
\left[x_{1}, x_{2}, \ldots, x_{n+1}\right] \equiv\left[\lambda x_{1}, \lambda x_{2}, \ldots, \lambda x_{n+1}\right] .
$$

In the remainder of this text homogeneous coordinates will be represented with square brackets whereas Cartesian coordinates will be denoted with parentheses.

The spaces $\mathbb{R} \mathbb{P}^{n}$ and $\mathbb{R}^{n}$ can be related to each other by defining the standard inclusion maps $i_{n}$ and standard projection maps $p_{n}$

$$
\begin{aligned}
& i_{n}: \mathbb{R}^{n} \rightarrow \mathbb{R P}^{n},\left(x_{1}, x_{2}, \ldots, x_{n}\right) \rightarrow\left[x_{1}, x_{2}, \ldots, x_{n}, 1\right] \\
& p_{n}: \mathbb{R P}^{n} \backslash P_{\infty}^{n} \rightarrow \mathbb{R}^{n},\left[x_{1}, x_{2}, \ldots, x_{n}, x_{n+1}\right] \rightarrow\left(\frac{x_{1}}{x_{n+1}}, \frac{x_{2}}{x_{n+1}}, \ldots, \frac{x_{n}}{x_{n+1}}\right),
\end{aligned}
$$


where $P_{\infty}^{n}$ is the set of points at infinity

$$
P_{\infty}^{n}=\left\{\left[x_{1}, x_{2}, \ldots, x_{n}, x_{n+1}\right] \in \mathbb{R P}^{n} \mid x_{n+1}=0\right\} .
$$

It is easily seen that $i=p^{-1}$ and vice versa, by the definition of homogeneous coordinates.

Finally, define the composition map $r_{p} \equiv i_{3} \circ r \circ p_{2}$ that takes the projective plane to the projective 3 -space

$$
\begin{aligned}
& r_{p}: \mathbb{R P}^{2} \rightarrow \mathbb{R P}^{3}, \\
& {\left[x_{1}, x_{2}, x_{3}\right] \rightarrow\left[\frac{\frac{4 x_{1}}{x_{3}}}{\left(\frac{x_{1}}{x_{3}}\right)^{2}+\left(\frac{x_{2}}{x_{3}}\right)^{2}+4}, \frac{\frac{4 x_{2}}{x_{3}}}{\left(\frac{x_{1}}{x_{3}}\right)^{2}+\left(\frac{x_{2}}{x_{3}}\right)^{2}+4}, \frac{\left(\frac{x_{1}}{x_{3}}\right)^{2}+\left(\frac{x_{2}}{x_{3}}\right)^{2}-4}{\left(\frac{x_{1}}{x_{3}}\right)^{2}+\left(\frac{x_{2}}{x_{3}}\right)^{2}+4}, 1\right]} \\
& \equiv\left[4 x_{1} x_{3}, 4 x_{2} x_{3}, x_{1}^{2}+x_{2}^{2}-4 x_{3}^{2}, x_{1}^{2}+x_{2}^{2}+4 x_{3}^{2}\right] \text {. }
\end{aligned}
$$

Notice that this mapping is given by a quadratic polynomial function.

\subsection{Rational Bézier patches}

A Bézier curve in $\mathbb{R} \mathbb{P}^{n}$ is defined as

$$
B(t)=\sum_{i=0}^{k} N_{i}^{k}(t) P_{i}
$$

where $P_{i}$ are called projective control points and

$$
N_{i}^{k}(t)=\left(\begin{array}{c}
k \\
i
\end{array}\right) t^{i}(1-t)^{k-i}
$$

is the Bernstein polynomial basis of degree $k$ over the interval $t=[0,1]$ [4]. A Bézier surface of degree $k$ by $l$ is obtained by taking the tensor product of two curves as follows

$$
B(u, v)=\sum_{i=0}^{k} \sum_{j=0}^{l} N_{i}^{k}(u) N_{j}^{l}(v) P_{i j},
$$

with $(u, v) \in[0,1] \times[0,1]$. Applying the standard projection to this surface leads to a rational Bézier surface $R \subset \mathbb{R}^{n}$. Taking $P_{i j}=\left[w_{i j} x_{i j}, w_{i j} y_{i j}, w_{i j} z_{i j}, w_{i j}\right]$,

$$
R(u, v)=\frac{\sum_{i=0}^{k} \sum_{j=0}^{l} N_{i}^{k}(u) N_{j}^{l}(v) w_{i j}\left(x_{i j}, y_{i j}, z_{i j}\right)}{\sum_{i=0}^{k} \sum_{j=0}^{l} N_{i}^{k}(u) N_{j}^{l}(v) w_{i j}} .
$$

Note that Bézier patches are homogeneous functions of the projective control points; scaling all projective control points by a factor $\lambda$ leaves the patch unchanged. Strictly speaking, a set of control points is therefore not just a set of points in $\mathbb{R} \mathbb{P}^{n}$, but rather a single point in $\left(\mathbb{R} \mathbb{P}^{n}\right)^{k l}$. 
Rational Bézier patches (and, more general, NURBS patches) have found widespread use in CAD software. One of the reasons for this is that these patches allow designers to exactly model quadrics, e.g. circles. In particular, it is possible to model a circular arc with a quadratic rational Bézier curve. Since Bézier surfaces are found as a tensor product of curves, it follows that a convex plane patch bounded by circle arcs can be represented by a biquadratic rational Bézier surface. We stress that convexity is an important property: if the (projected) patch is nonconvex, the circular curves defined by the outer control points may not coincide with the real boundary of the patch. The mapping is not injective in that case. See Fig. 3 for an illustration of this effect.

\subsection{Generating spherical tiles as Bézier patches}

The elements discussed above are now combined in a general methodology for finding the Bézier representation of a quadrilateral spherical tile. Consider a tile that is defined by the four vertices $V_{i} \in \Sigma, i=1, \ldots, 4$. An edge is formed by connecting two vertices with a great circle arc. The great circle $\Gamma$ itself is then given by the intersection of the unit sphere with the plane containing the origin and the two vertices,

$$
\Gamma=\{(x, y, z) \in \Sigma \mid a x+b y+z=0\},
$$

where it is first assumed that the $z$-axis is not in this plane. The stereographic projection $(u, v)=s(x, y, z)$ of this curve satisfies the circle equation

$$
(u+2 a)^{2}+(v+2 b)^{2}=4\left(1+a^{2}+b^{2}\right),
$$

which can be verified by simply applying the definition (2) to points on $\Gamma$ - see Fig. 2 for illustration. Hence, the projected edge is also a circular arc with endpoints given by the projected vertices $\widehat{V}=s(V)=\left(2 x_{V}, 2 y_{V}\right) /\left(1-z_{V}\right)$ and centre given

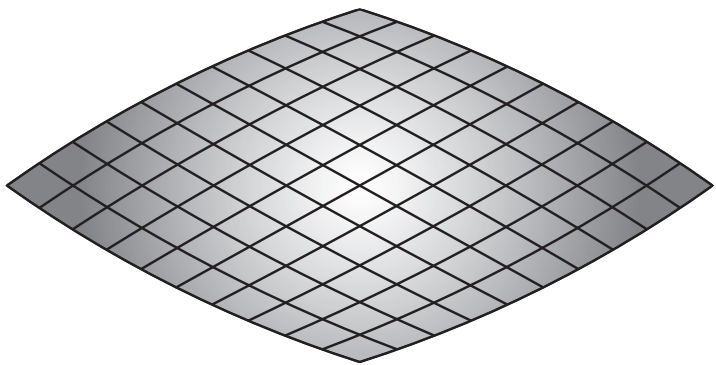

(a)

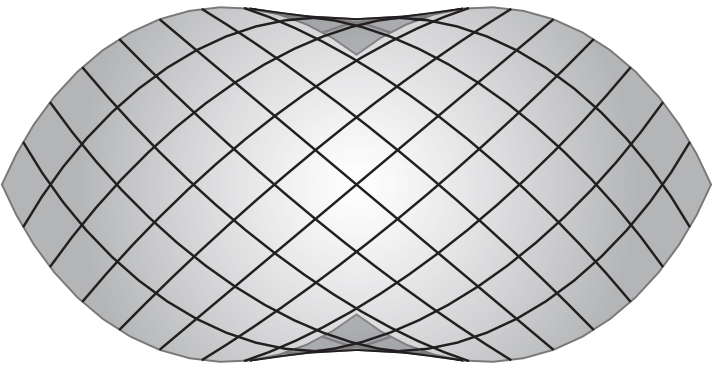

(b)

Figure 3: A convex (a) and nonconvex (b) patch bounded by circle arcs that respectively can and cannot be represented as a Bézier patch. 
by $C=(-2 a,-2 b)$. If one defines the midpoint $M=\left(\widehat{V}_{1}+\widehat{V}_{2}\right) / 2$ and the factor $\alpha=|M-C| /\left(2 \sqrt{1+a^{2}+b^{2}}\right)$, the projective control points for such an arc are given by the expressions [4]

$$
\widehat{P}_{1}=\left[\widehat{V}_{1}, 1\right], \widehat{P}_{2}=\left[C+\frac{(M-C)}{\alpha^{2}}, \alpha\right], \widehat{P}_{3}=\left[\widehat{V}_{2}, 1\right]
$$

where it is understood that these points are defined up to a common scaling factor. The indices in the above equation have been made explicit for clarity, but of course the reasoning itself applies to all arcs.

In case the plane defining $\Gamma$ does contain the $z$-axis,

$$
\Gamma=\{(x, y, z) \in \Sigma \mid a x+b y=0\},
$$

the result is simplified since the stereographic projection of such a great circle becomes a line - see Fig. 2 - with equation

$$
a u+b v=0 .
$$

For the control points, one can now take

$$
\widehat{P}_{1}=\left[\widehat{V}_{1}, 1\right], \widehat{P}_{2}=[M, 1], \widehat{P}_{3}=\left[\widehat{V}_{2}, 1\right] .
$$

Since Bézier patches are interpolatory at their edges, it follows that the control points obtained with the above method can be used to define the boundary of the projected tile. Ultimately this leaves a single point (the central control point) undefined. There is some freedom in choosing this point: as long as it lies in between the outer points it tends to affect the parameterization, but not the geometry of the surface. In this work, the central control point is chosen to be the mean of the outer points. The motivation is that this choice should help preserve some of the geometric symmetry in the resulting mesh.

Remark. It is clear from the above discussion that the parameterization of the projected patch is not unique. The position of the central control point is always free (to a certain extent). More importantly, if any edges of the patch happen to be line segments, they also have multiple quadratic Bézier parameterizations. This ultimately affects the control mesh along the edge of the spherical tile and therefore determines whether or not the mesh is conforming.

As it stands, a Bézier representation of the stereographically projected sphere tile $\widehat{T}$ has been obtained:

$$
\widehat{T}(u, v)=\sum_{i=0}^{2} \sum_{j=0}^{2} N_{i}^{2}(u) N_{j}^{2}(v) \widehat{P}_{i j} .
$$


The map $\widehat{T}$ is a biquadratic function in $(u, v)$ and $\widehat{P}_{i j}$ is a three-by-three projective control net. The only remaining task is to transform this surface into a tile of the sphere, which is done by simply applying the inverse stereographic projection

$$
T(u, v) \equiv r_{p} \circ \widehat{T}(u, v) .
$$

Since this map is the composition of two quadratic polynomial functions, it must be a quartic polynomial in $(u, v)$. Therefore, the tile itself can be represented in the quartic Bernstein basis

$$
T(u, v)=\sum_{i=0}^{4} \sum_{j=0}^{4} N_{i}^{4}(u) N_{j}^{4}(v) P_{i j} .
$$

Remark. The procedure outlined here generally leads to a biquartic rational Bézier parameterization. Dietz et al. [29, 30] showed that, on the sphere, a quadrangular patch bounded by circle segments has a biquadratic Bézier parameterization if and only if the sum of the internal angles equals $2 \pi$. For quadrilateral spherical polygons (bounded by great circles) this sum is always strictly larger than $2 \pi$, hence no biquadratic form exists. Interestingly, it is also shown in [29] that any quadrilateral polygon has a representation of degree $(2,4)$. In the present paper this possibility is not further investigated since the mixed degree would break some of the symmetry and hence the ability to make conforming meshes.

The problem of finding the five-by-five projective control net $P_{i j}$ can be solved in at least two different ways. One possibility is to explicitly calculate the map $T$, expanding it into its terms and then converting the result to the Bernstein basis. The control point values can then be simply read off. For the tilings generated here, however, a simple collocation approach is used: 25 points $(u, v)$ are taken and their images under the inverse stereographic map are computed. Enforcing these images gives 25 vector equations for the control points, yielding a uniquely determined linear system that is readily solved. A MATLAB implementation of the above procedure is made available in the supplementary material of this manuscript $2^{2}$

\section{Spherical tilings}

There exists an infinite variety of ways to tile the sphere, but the possibilities are strongly restricted by some practical and mathematical considerations.

- Bézier surfaces have a tensor product structure, inherently limiting their ability to represent non-quadrilateral tiles.

\footnotetext{
${ }^{2}$ This implementation has also been integrated in the Octave NURBS Toolbox [31. It is to be released in version 1.3.14.
} 
- Certain degeneracies in the geometric mapping are to be avoided. In particular, any tiles that have $180^{\circ}$ angles between adjacent sides or any tiles where sides collapse to points are prohibited.

- The tiles should be geodesically convex to avoid 'spilling' across the edges (see 2.3).

- For the sake of brevity, only monohedral tilings are considered here. The complete tessellation of the sphere can then be obtained by successive rotations of a single tile about the sphere's centre.

The central projections of the Platonic solids [32] (Fig. 4) form an obvious group of candidate geometries, because they have a very high level of symmetry. The spherical cube is the only one of these that directly yields quadrangular tiles, making it the simplest pattern. The cube topology was already treated by Cobb [5] and is shown in Fig. 5a. Nonetheless, the other shapes are also useful. For example, equilateral triangular patches can be joined pairwise to form rhombi. Applying this operation to the spherical octahedron yields a hosohedral tiling, consisting of four degenerate rhombi. If the spherical tetrahedron is used as a starting point, the 'obtuse' angles become larger than $180^{\circ}$ so the rhombi are not geodesically convex. It is clear that neither of these are in line with the requirements of the last paragraph. The only remaining candidate is the paired icosahedral tiling, made up of 10 tiles meeting at 12 vertices (2 pentavalent and the others trivalent). This tiling is depicted in Fig. 5b.

Aside from the tessellations based on the Platonic solids, there exist other possibilities to tile the sphere. For instance, the paired icosahedral tiling is only a single member of an infinite family of rhombic tilings, all topologically equivalent to trapezohedra. Alternatively, one can relax some of the symmetry restrictions imposed on the Platonic solids, namely the restrictions of isogonality (vertextransitivity) and isotoxality (edge-transitivity). The tilings obtained in this fashion are related to the so-called semiregular polyhedra. In this treatment, only those polyhedra that are still face-transitive are considered. These shapes are known as Catalan solids [32]. Two members of this class have rhombic faces: the rhombic
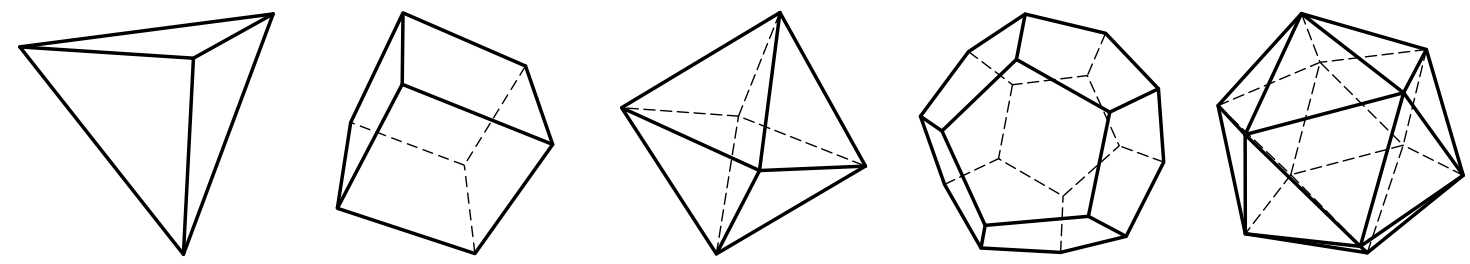

Figure 4: The Platonic solids. From left to right: tetrahedron, hexahedron (cube), octahedron, dodecahedron and icosahedron. 
dodecahedron (Fig. 5c) and the rhombic triacontahedron (Fig. 5d). Incidentally, these are also the only two edge-transitive Catalan solids [32]. The rhombic dodecahedron is a shape with 12 faces and 24 edges, 8 trivalent and 6 tetravalent vertices. The rhombic triacontahedron has 30 faces and 60 edges. Its faces are joined in 20 trivalent and 12 pentavalent vertices. The two other Catalan solids with quadrangular faces are the deltoidal icositetrahedron and the deltoidal hexecontahedron. (Figs. 5e 5f). Instead of rhombi, the faces of these two shapes are right-angled kites (deltoids). The deltoidal icositetrahedron consists of 24 faces separated by 48 edges, 8 trivalent and 18 tetravalent vertices - the tetravalent vertices belong to two distinct classes, see Fig. 5e. Finally, the deltoidal hexecontahedron has 60 faces and 120 edges in total. Altogether there are 20 trivalent, 30 tetravalent and 12 pentavalent vertices. It is interesting to see that both deltoidal Catalan tilings result from the combination of two dual tilings: the spherical cube and octahedron together yield the deltoidal icositetrahedron, whereas the deltoidal hexecontahedron is the combination of the spherical dodecahedron and icosahedron. Similarly, the rhombic dodecahedron can be described by two dual tetrahedra.

More quadrilateral tilings might be designed - for an exhaustive classification, see 33 - but the cases given above form a representative sample of the possible convex monohedral tilings. If desired, the reader can use the method from Section 2.4 to generate tilings meeting his own criteria. The further purpose of the present paper is not to give a comprehensive overview of the possible tilings but to study effects on multipatch geometry descriptions in IGA. In this context, the parameterization of the tiling is of considerable importance, determining mesh conformity across patches. In Section 2.4, it was shown that some edges of the projected patch may become straight, allowing more than one parameterization. The extra freedom might be used to generate conforming meshes for certain topologies, but at present it is unclear how manipulations of the projected tile's control net affect the associated spherical tile; this could be a topic for further investigation. In the present study we chose to avoid the complication by prohibiting tiles whose edges become line segments after the stereographic projection. For the five novel multipatch sphere representations described here, this approach has led to meshes that are both conforming and nonconforming. More specifically, conforming meshes have been found for all topologies except the paired icosahedron. In fact, it shows a very interesting type of nonconformity: both the geometry and the knot vectors are the same on two adjacent patches, yet the parameterization is not. We will henceforth call these meshes intrinsically nonconforming. The control nets representing the novel tilings (and, for convenience, the cube topology) are documented in Appendix A. An electronic version of this data can be found in the 


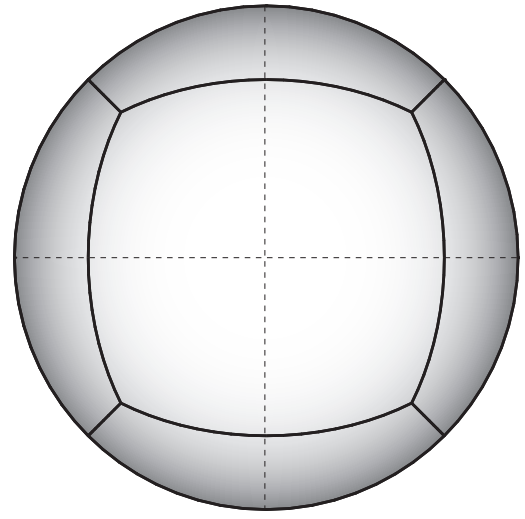

(a) Cube

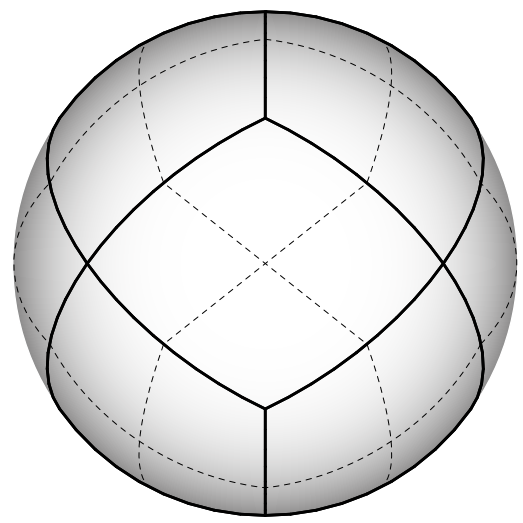

(c) Rhombic dodecahedron

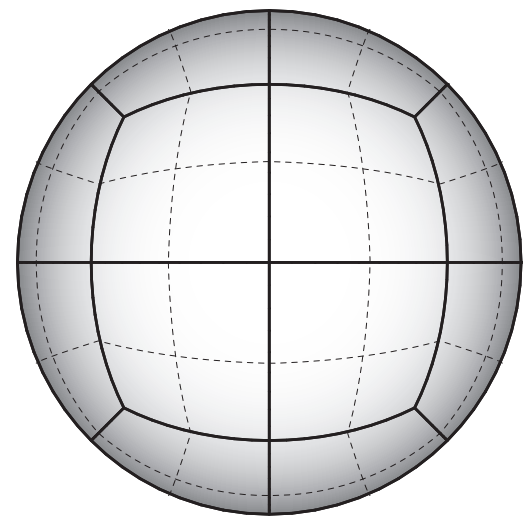

(e) Deltoidal icositetrahedron

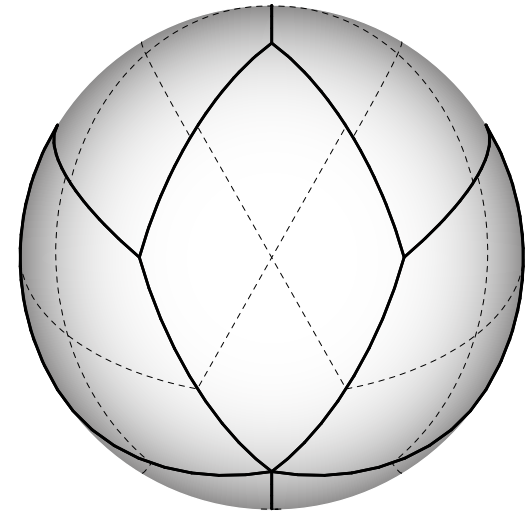

(b) Paired icosahedron

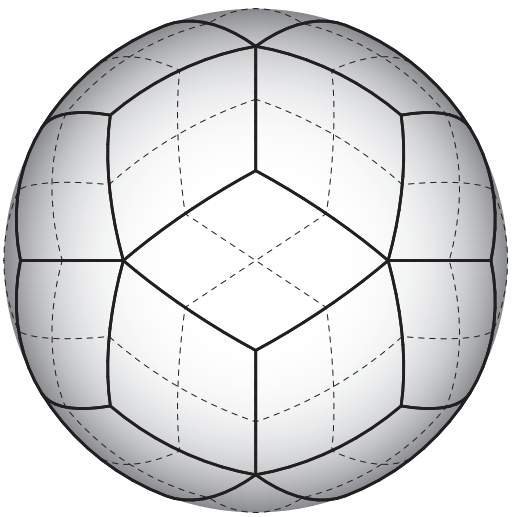

(d) Rhombic triacontahedron

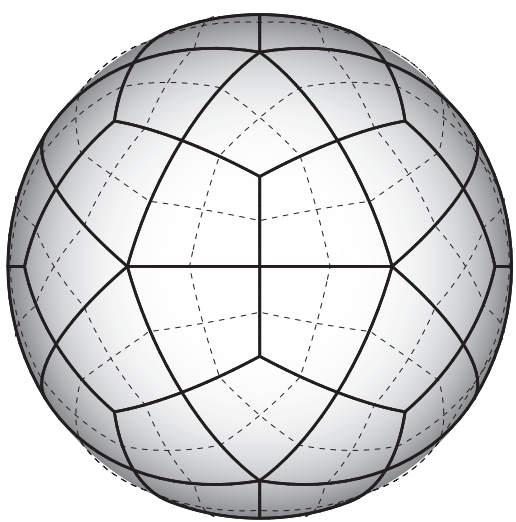

(f) Deltoidal hexecontahedron

Figure 5: Spherical tilings derived from the Platonic (a-b) and Catalan (c-f) solids with internal mesh lines. Note the nonconformity in the paired icosahedral tiling (b). 
supplementary material of this text $:^{3}$

\section{Numerical case studies}

The spherical tilings resulting from the above development are now integrated in existing IGA codes for the treatment of three case studies involving spherical geometries. The main goal is to introduce these problems as geometrically nontrivial benchmarks for comparing $C^{0}$ and/or $C^{1}$-multipatch IGA methodologies. To this end, a brief theoretical description is made and the relevant literature is given for each of these problems. In addition, either the analytic expressions or accurate numerical approximations of the solutions are included for reference.

As a preliminary, it is useful to study the regularity and distortion of the geometric maps, since this may give an indication of the analysis-suitability of the employed sphere parameterizations and help in interpreting the obtained results. We define three local quality measures here, in analogy to common finite element quality parameters. Let the tangent vectors along the $u$ and $v$ directions be denoted $t_{u}$ and $t_{v}$ respectively. Then the local normalized scale factor $\phi$, local skewness $\sigma$ and local aspect ratio $\alpha$ are defined as:

$$
\phi=\frac{\left\|t_{u} \times t_{v}\right\|}{\gamma}, \quad \sigma=1-\frac{\left\|t_{u} \times t_{v}\right\|}{\left\|t_{u}\right\|\left\|t_{v}\right\|}, \quad \alpha=\max \left\{\frac{\left\|t_{u}\right\|}{\left\|t_{v}\right\|}, \frac{\left\|t_{v}\right\|}{\left\|t_{u}\right\|}\right\},
$$

where $\gamma$ is the patch area. For instance, the scale factor - analogous to the Jacobian determinant - should remain strictly positive everywhere to ensure an injective parameterization which is suitable for analysis [34]. To the best of our knowledge, the effects of skewness and aspect ratio have not yet been studied in IGA applications, but these factors are included here because they give a fairly detailed picture of the map distortions. In a global sense, then, the quality might be measured by the maximum skewness, the maximum aspect ratio and the ratio between the maximal and minimal scale factors - for an equidistant square mesh these values would become 0,1 and 1 respectively. Fig. 6 shows the local and global quality measures for each of the tilings discussed in the previous section. The tilings are compared to a classical representation with a polar singularity - i.e. obtained by revolving a semicircle. According to these measures, all tilings seem quite well-behaved. In contrast: for the classical parameterization the global quality measures reach extremely bad values. The maximal skewness becomes one, and both the maximum aspect ratio and the scale factor ratio become infinitely large due to the singular points. Even though isogeometric analysis was shown to be more robust to

\footnotetext{
${ }^{3}$ This code has also been integrated in the Octave NURBS Toolbox [31]. It is to be released in version 1.3.14.
} 
these flaws [35], the parameterization still influences the accuracy of the obtained solution [34, 36, 40].

\subsection{Plane wave scattering by a rigid sphere}

In this section the scattering of an acoustic plane wave by a rigid sphere suspended in an acoustic medium is considered, as an example of a problem requiring $C^{0}$-continuity. If a plane pressure wave of unit amplitude and wavenumber $k$ impinges on a sphere of radius $R$, the resulting scattered acoustic pressure field $\widehat{p}_{s}(r, \theta)$ is given analytically as [41, 42,

$$
\widehat{p}_{s}(r, \theta)=\sum_{n=0}^{\infty}-(2 n+1) \mathrm{i}^{n} \frac{(n+1) j_{n+1}(k R)-n j_{n-1}(k R)}{(n+1) h_{n+1}^{(2)}(k R)-n h_{n-1}^{(2)}(k R)} P_{n}(-\cos \theta) h_{n}^{(2)}(k r),
$$

with $r$ the radial distance from the sphere's centre and $\theta$ the accompanying polar angle (measured with respect to the plane wave propagation direction). The function $P_{n}$ is the Legendre polynomial, $j_{n}$ the spherical Bessel function of the first kind, and $h_{n}^{(2)}$ the spherical Hankel function of the second kind, all of order $n$. The imaginary unit is represented by the symbol $\mathrm{i}$.

Consider here the same instance of the problem as [13]. A monochromatic plane wave of frequency $f=100 \mathrm{~Hz}$, propagating in air with density $\rho=1.225 \mathrm{~kg} \mathrm{~m}^{-3}$ and wave velocity $c=340 \mathrm{~m} \mathrm{~s}^{-1}$, is scattered by a sphere of radius $1 \mathrm{~m}$. The analytic solution to this problem is shown in Fig. 7. As the problem treats acoustics, the natural solution domain is the three-dimensional space. However, digital geometric designs are fundamentally surfaces, so to accommodate this problem in the framework of IGA it is convenient to convert the solution domain to a surface. This remark is relevant to the present study in particular, since we have developed discretizations of a spherical surface. Hence, the numerical simulations are carried out using a boundary element method (BEM) [43, 44]. The isogeometric indirect boundary element formulation for use in exterior acoustics described in [45] is used here.

Spherical meshes of different topologies and refinements are used to perform the numerical analysis. The accuracy of the boundary element simulation is assessed by the relative $L^{2}$-norm error $\left\|p_{s}-\widehat{p}_{s}\right\|_{2} /\left\|\widehat{p}_{s}\right\|_{2}$ on the surface pressure, where the standard definition of the $L^{2}$-norm

$$
\|\bullet\|_{2}=\sqrt{\int_{\Omega}|\bullet|^{2} d \Omega}
$$

applies. The characteristic element length scale $h$, defined as the square root of the average area of an element, is used for comparison. Depending on the topology used, different levels of accuracy are obtained for elements of the same 

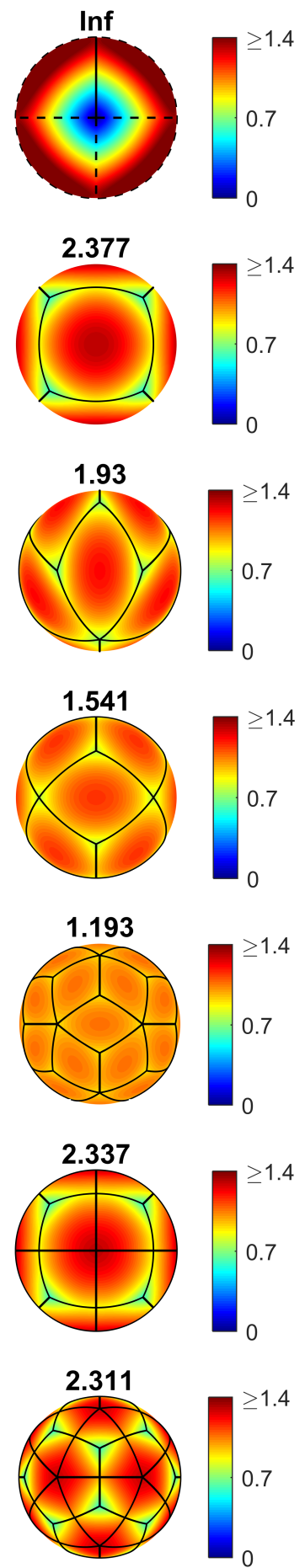
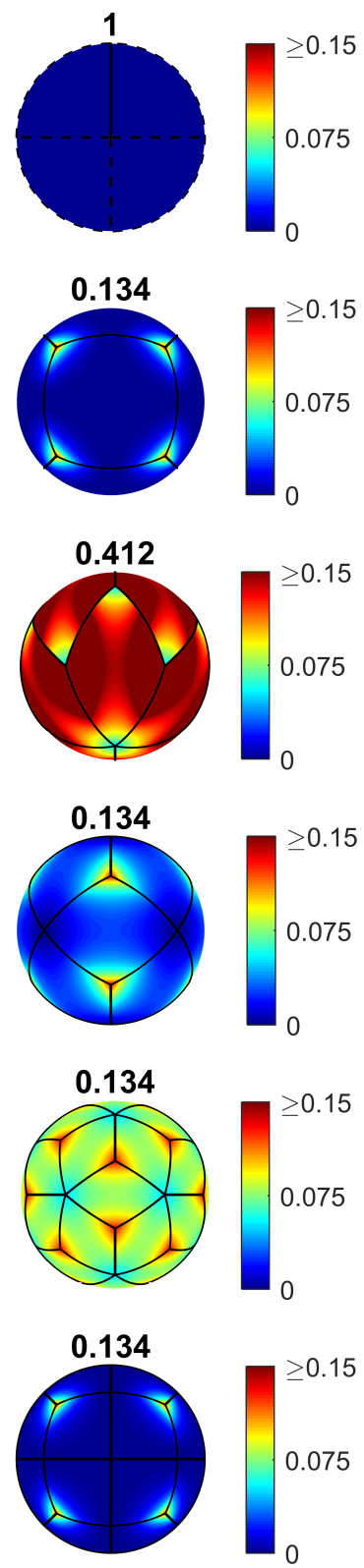

$$
0.134
$$

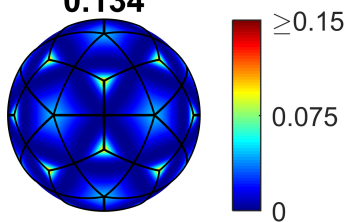

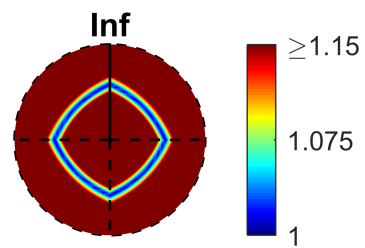
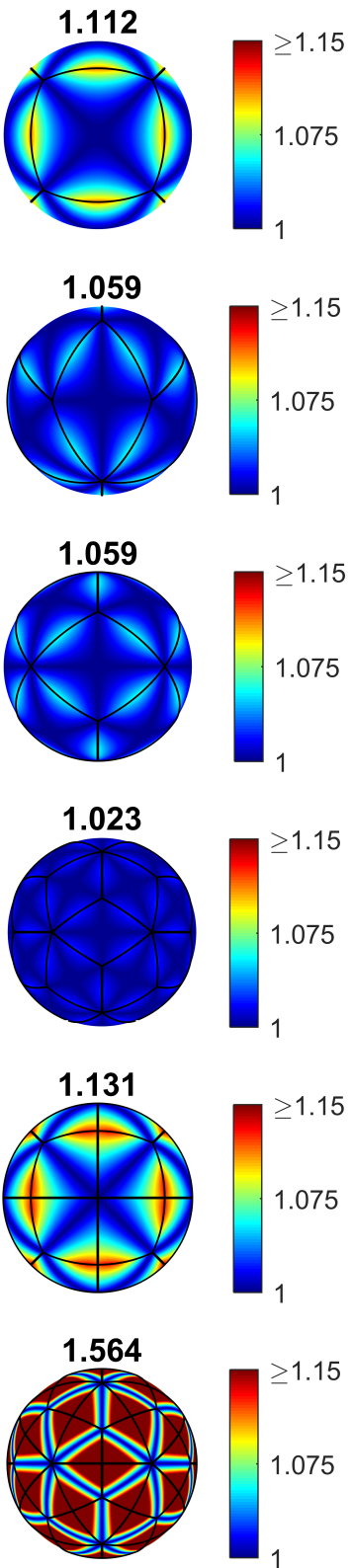

Figure 6: Quality measures of the parameterizations for the classical representation (in polar view) and the spherical tilings. From left to right: scale factor (ratio), skewness (maximum), aspect ratio (maximum). 


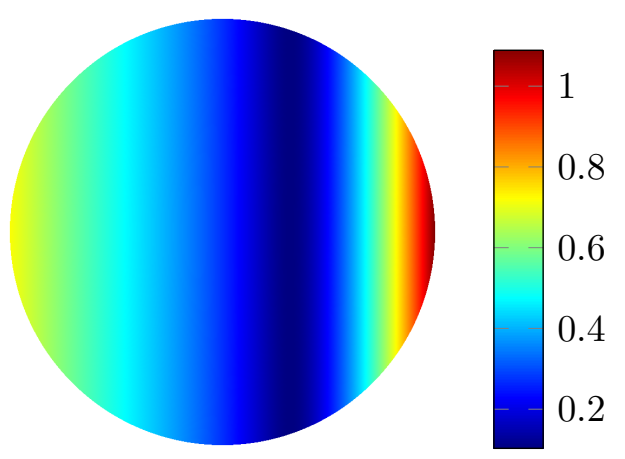

Figure 7: Colour plot of the analytical scattered pressure field [Pa] on the sphere's surface (Eq. (14)). The impinging plane wave is travelling from left to right. It has $f=100 \mathrm{~Hz}$ and is propagating in air with density $\rho=1.225 \mathrm{~kg} \mathrm{~m}^{-3}$ and wave velocity $c=340 \mathrm{~m} \mathrm{~s}^{-1}$. It is scattered by a sphere with $R=1 \mathrm{~m}$.

size. First the conforming meshes are considered, all indicated in Fig. 8. The observed convergence rate is about the same for each topology. However, because of the initial sharp drop in the error, both deltoidal meshes strongly outperform all others. Although these meshes have a larger DOF density for a given mesh size (because of the additional basis functions introduced near the interfaces), such large discrepancy in performance is unexpected.

The nonconforming mesh of the paired icosahedral tiling leads to a more difficult test for patch coupling methods. In general, the coupling of nonconforming patches is not trivial even in the $C^{0}$-case [13]. Moreover, an extra complication arises because the nonconformity is intrinsic. This means that coupling techniques acting in the parametric domain (for instance, collocation in the Greville abscissae) may become less effective or even useless since the images of a specified (parametric) boundary point may not coincide for two adjacent patches. In fact, the simulations of this case led us to discover a shortcoming in the VUKIMS [13] patch coupling routine. Initially, the code failed to recognize the intrinsic nonconformity, preventing convergence to the correct solution. However, we found that convergence can be obtained by applying collocation in the physical points corresponding to the Greville abscissae of both sides of the interface.

\subsection{Static loading of a pinched hemispherical shell}

The next problem is taken from the 'shell obstacle course' proposed by Belytschko et al. [46] and inspired by the earlier work of MacNeal \& Harder [47]. It considers the elastostatic deflection of an hemispherical shell. The shell is loaded with four radial point forces of magnitude $F=2 \mathrm{~N}$, spaced over $90^{\circ}$ and alternating in sign (see Fig. 9). It has a midsurface radius $R=10 \mathrm{~m}$ and thickness $t=0.04 \mathrm{~m}$, and consists of a homogeneous isotropic material with Young's modulus $E=6.825 \times 10^{7} \mathrm{~Pa}$ and Poisson's ratio $\nu=0.3$. Despite the fairly large 


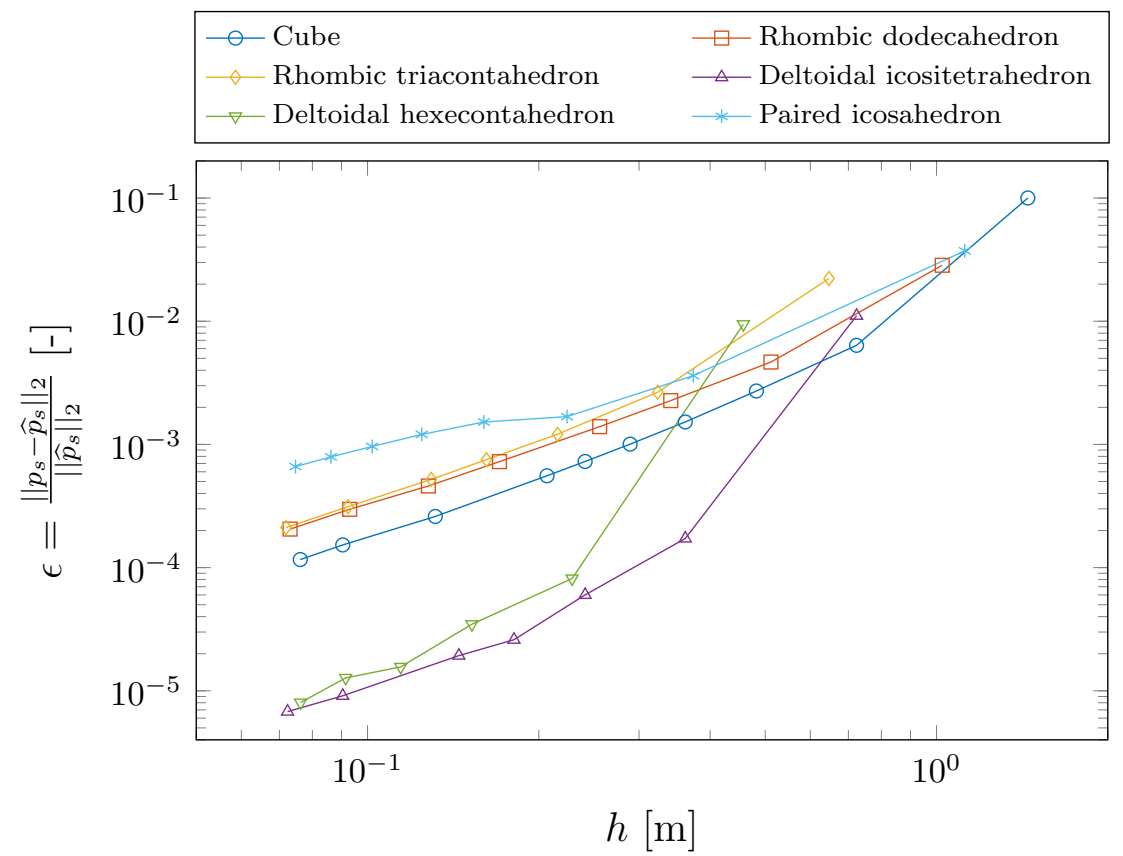

Figure 8: Relative $L^{2}$-norm error of the surface pressure field versus the element dimension for the scattering sphere problem. The paired icosahedral tiling has an intrinsically nonconforming mesh, whereas all the others are conforming

resulting deformations, the linear theory of elasticity is applied in this model [46]. The studied benchmark quantity is the radial displacement $w_{F}$ at the application point of one of the forces. In [46], a value $w_{F}=0.0924 \mathrm{~m}$ is mentioned for this case. This displacement is an approximation (lower bound) obtained using the Rayleigh-Ritz method with trigonometric trial functions [48].

Taking advantage of the symmetry, it suffices to model only a quarter of the domain (namely an octant of the sphere). The resulting problem has already been studied in IGA by Cottrell et al. [3], but in their work a single degenerate NURBS patch is used to describe the octant. The deltoidal icositetrahedral tiling described above (Fig. 5e provides an alternative description. It perfectly divides the sphere into octants and has the benefit of being degeneracy-free. However, since this is a multipatch discretization, a patch coupling technique needs to be supplied for analysis. In the present study, the rotation-free Kirchhoff-Love shell formulation introduced by Kiendl et al. [49] is used to describe the deformations. This shell formulation requires the displacement fields to be $C^{1}$-continuous. In order to impose this condition between the three conforming patches, the collocation method from [27] is used here. To assess the performance of the multipatch approach, two single-patch discretizations are studied as well. Both are degenerate, but to different degrees. The first patch has a degenerate mapping, since one of its edges 


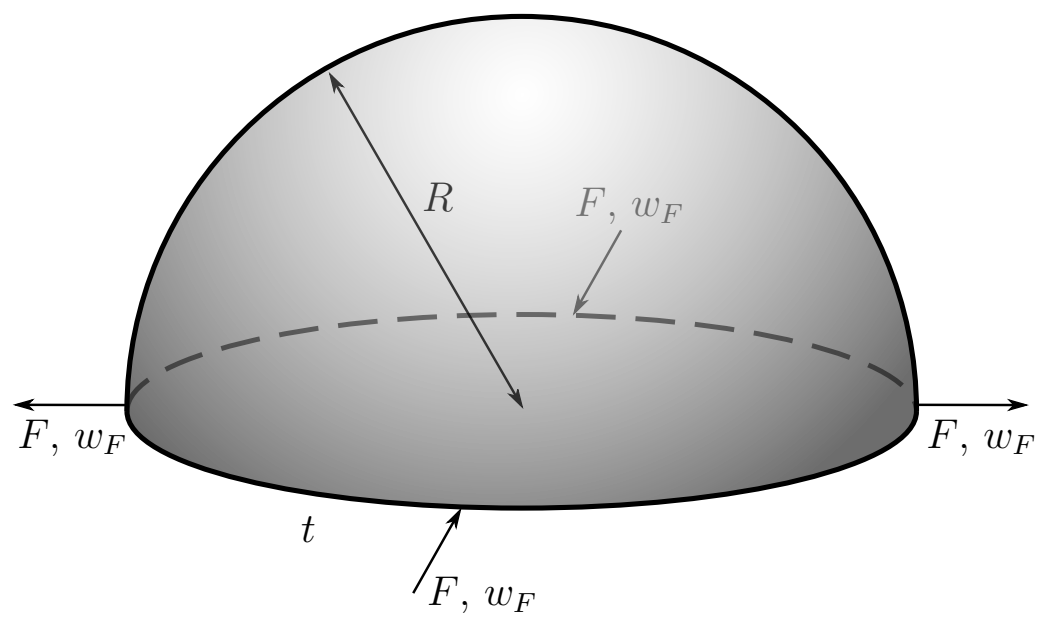

Figure 9: The pinched hemispherical shell.

is collapsed to a point; the second patch has a degenerate derivative because one of its internal angles is straight. Fig. 10 depicts all three discretizations. The displacement field on the reduced domain is shown as a colour plot in Fig. 11.

In Fig. 12, the convergence of the numerical method is plotted. We note that, for the sake of precision, the errors have been computed with respect to a reference value $\widehat{w}_{F}=0.0924129 \mathrm{~m}$, which was obtained using the finest mesh and which is consistent with the (less precise) solution from literature. Convergence is observed for all parameterizations. However, it appears that the introduction of patch coupling leads to a small performance drop that cannot be compensated entirely by the elimination of degeneracies. The degenerate parameterizations both achieve slightly faster convergence than the multipatch case.

\subsection{Vibrations of a spherical shell}

As a last example, the vibration of a thin spherical shell in vacuum is considered. One of the earliest treatments of this problem [50] precedes even the thin-shell theory of Love [51]. In the subsequent decades, some special cases of the spherical shell were further studied using Love's theory [52 55$]$ - see [56] for a good overview up to the late sixties. Finally, Niordson [57, 58] gave a general description that includes nonaxisymmetric vibration modes. It is shown there that, depending on the value of the angular frequency $\omega$ with respect to a critical value, the vibration can be in one of two different regimes. The low- and high-frequency cases are treated in [57] and [58] respectively. We limit ourselves to the low-frequency regime in the present paper.

Consider a complete spherical shell with midsurface radius $R$ and thickness $t$ with $t \ll R$ that is freely vibrating. The shell consists of a homogeneous and isotropic material with Young's modulus $E$, Poisson's ratio $\nu$ and density $\rho$. In 

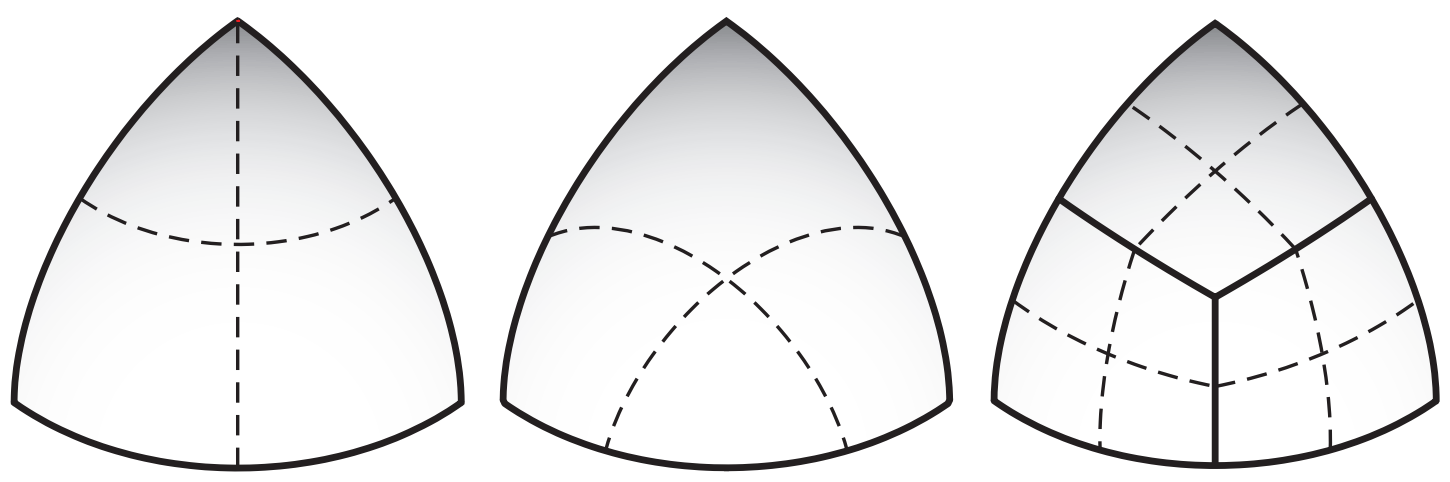

Figure 10: Three different parameterizations of a spherical octant. From left to right: Singlepatch parameterization with a degenerate edge, single-patch parameterization with a degenerate vertex, multipatch parameterization (degeneracy-free). Dashed lines show internal knot lines.

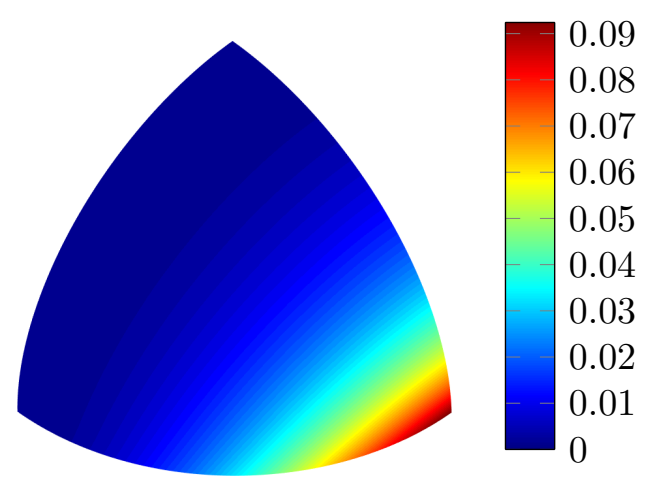

Figure 11: Colour plot of displacements $[\mathrm{m}]$ for an hemispherical shell under a pinching load (Fig. 9p. The parameters are as follows: $F=2 \mathrm{~N}, R=10 \mathrm{~m}, t=0.04 \mathrm{~m}, E=68.25 \mathrm{MPa}$ and $\nu=0.3$. The component of displacement along the axis of one of the forces is plotted. The maximum (calculated numerically) is $\widehat{w}_{F}=0.0924129 \mathrm{~m}$.

accordance with the early findings of Lamb [50], Niordson's derivation leads to two solution classes [57]. For the first class, the eigenfrequencies are given by

$$
\omega=\sqrt{(n-1)(n+2)} \frac{1}{R} \sqrt{\frac{E}{2 \rho(1+\nu)}} .
$$

The corresponding eigenmodes describe purely tangential displacements. On the other hand, the frequencies of the second class are found as the positive roots of the characteristic equation

$$
\begin{array}{r}
\bar{\omega}^{4}-\bar{\omega}^{2}\left\{1+3 \nu+\lambda-\beta^{2}\left[-\lambda^{2}+\lambda(\nu+3)-2(1+\nu)\right]\right\} \\
+(\lambda-2)\left(1-\nu^{2}\right)+\beta^{2}\left[\lambda^{3}-4 \lambda^{2}+\lambda\left(5-\nu^{2}\right)-2\left(1-\nu^{2}\right)\right]=0,
\end{array}
$$




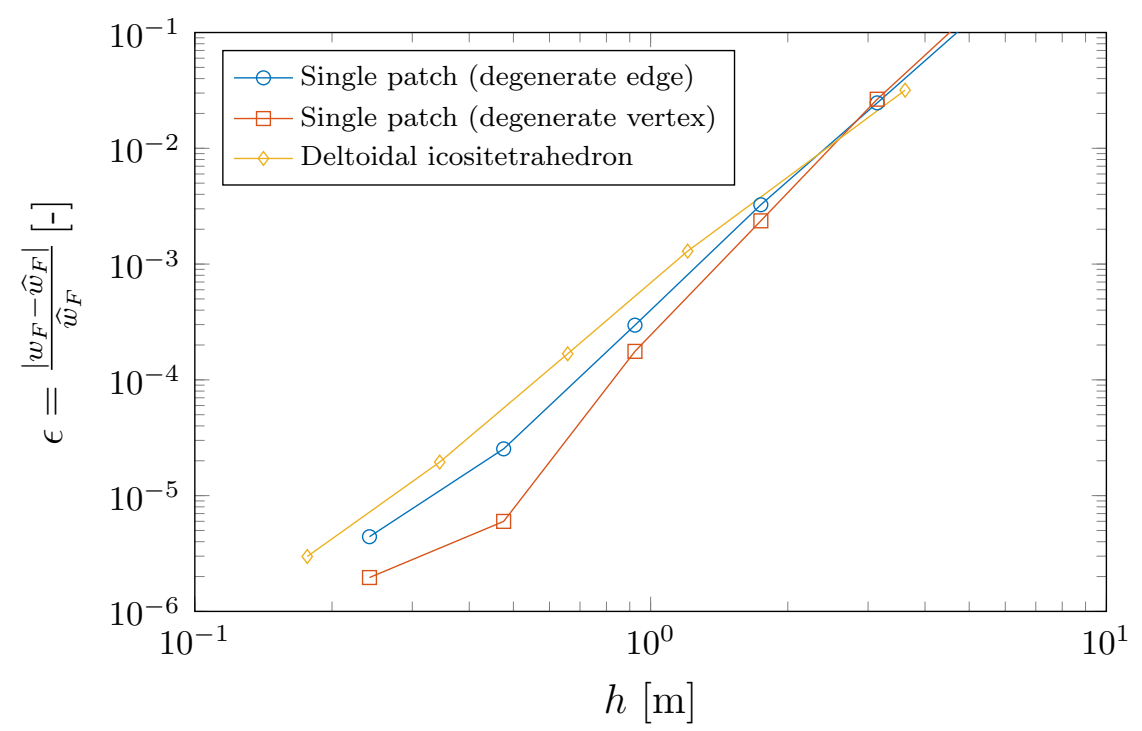

Figure 12: Convergence of the radial displacement $w_{F}$ versus characteristic element size $h$. Errors are computed with respect to the most precise numerical solution $\widehat{w}_{F}=0.0924129 \mathrm{~m}$.

where $\bar{\omega}=\omega R \sqrt{\rho\left(1-\nu^{2}\right) / E}$ is a dimensionless frequency, $\beta=t /(\sqrt{12} R)$ is a parameter indicating the slenderness and $\lambda=n(n+1)$. The solutions are given by

$$
\bar{\omega}=\sqrt{\frac{\left\{1+3 \nu+\lambda-\beta^{2}\left[-\lambda^{2}+\lambda(\nu+3)-2(1+\nu)\right]\right\} \pm \Delta}{2}},
$$

with

$$
\begin{array}{r}
\Delta^{2}=\left\{1+3 \nu+\lambda-\beta^{2}\left[-\lambda^{2}+\lambda(\nu+3)-2(1+\nu)\right]\right\}^{2} \\
-4\left\{(\lambda-2)\left(1-\nu^{2}\right)+\beta^{2}\left[\lambda^{3}-4 \lambda^{2}+\lambda\left(5-\nu^{2}\right)-2\left(1-\nu^{2}\right)\right]\right\} .
\end{array}
$$

The modes corresponding to these frequencies have a finite transverse (radial) displacement. For $n=0$, there is only one real root. Associated with this frequency is the breathing mode, which is a uniform extension-compression of the shell. In the rest of the low-frequency regime, there are two positive roots.

In a seemingly independent derivation by Junger and Feit [42], only the axisymmetric modes are studied. They obtain the following characteristic equation:

$$
\begin{array}{r}
\bar{\omega}^{4}-\bar{\omega}^{2}\left[1+3 \nu+\lambda-\beta^{2}\left(1-\nu-\lambda^{2}-\nu \lambda\right)\right] \\
+(\lambda-2)\left(1-\nu^{2}\right)+\beta^{2}\left[\lambda^{3}-4 \lambda^{2}+\lambda\left(5-\nu^{2}\right)-2\left(1-\nu^{2}\right)\right]=0,
\end{array}
$$

which becomes identical to Eq. (17) for membranes $(\beta=0)$ but gives slightly different solutions for shells of nonzero thickness due to the difference in the coef- 
ficient of $\bar{\omega}^{2}$. Explicitly, the solutions of Eq. 20 are

$$
\bar{\omega}=\sqrt{\frac{\left[1+3 \nu+\lambda-\beta^{2}\left(1-\nu-\lambda^{2}-\nu \lambda\right)\right] \pm \Delta}{2}},
$$

with

$$
\begin{array}{r}
\Delta^{2}=\left[1+3 \nu+\lambda-\beta^{2}\left(1-\nu-\lambda^{2}-\nu \lambda\right)\right]^{2} \\
-4\left\{(\lambda-2)\left(1-\nu^{2}\right)+\beta^{2}\left[\lambda^{3}-4 \lambda^{2}+\lambda\left(5-\nu^{2}\right)-2\left(1-\nu^{2}\right)\right]\right\} .
\end{array}
$$

The reason for the discrepancy between Eqs. (17) and (20) is at present unclear to us.

It has furthermore been observed by different authors [55, 57] that there are multiple eigenmodes corresponding to each eigenfrequency. This degeneracy occurs because of the spherical symmetry of the problem. More precisely, there exist $2 n+1$ orthogonal modes for each frequency [55]. Rigid-body motion occurs when $n=1$, since then $\omega=0$ is a solution to any of Eqs. (16), (17) or 20). The triplets of rigid-body rotation and translation modes belong respectively to the first and second solution classes.

For our benchmark only one of the natural frequencies is studied. The lowest nonzero pulsation is selected, henceforth named $\omega_{0}$. Analytically, this pulsation is found as the smaller second-class frequency for the case $n=2$, i.e. the small solutions to Eqs. 18 19) or 21 22 with $\lambda=6$. In the cases considered, the parameters are set as follows: $R=1 \mathrm{~m}, E=70 \mathrm{GPa}, \nu=0.3$ and $\rho=2700 \mathrm{~kg} \mathrm{~m}^{-3}$. The problem is solved numerically using an isogeometric Kirchhoff-Love shell formulation [49], where the method proposed in [27] is employed to enforce $C^{1}$-continuity of the transverse displacements. For $C^{0}$-coupling of the intrinsically nonconforming mesh, the collocation approach mentioned in Section 4.1 is used.

In the limit $\beta \rightarrow 0$, both theoretical expressions are in agreement with each other. This limit physically corresponds to a vibrating membrane without flexural rigidity. Numerically, this behaviour is simulated by studying a Kirchhoff-Love shell for which the bending stiffness has been abstracted. Since the only remaining stresses are in-plane (membrane stresses), $C^{0}$-continuity is actually sufficient in this case, and in our simulations continuity was only enforced up to this level. Using the same parameters as before (with the exception of the slenderness), the analytic solution yields $\widehat{\omega}_{0}=3.7413348277488 \times 10^{3} \mathrm{~s}^{-1}$. This value is used as the reference value in Fig. 13a. All conforming mesh topologies lead to convergence up to ten significant digits, verifying the accuracy of our methodology for these cases. In addition, the simulations were carried out using a single-patch parameterization, obtained by revolving a semicircle. In this case, the convergence order is similar, but the errors are slightly larger. The convergence curve of the intrinsically nonconforming tiling, however, levels out after obtaining 6 digits of 
precision. We believe that this is due to the rudimentary $C^{0}$-coupling method that was employed (see Section 4.1). The development of a robust coupling method for intrinsically nonconforming meshes is a worthwhile topic for future research.

Next, a general case with $t=0.01 \mathrm{~m}($ or $\beta=1 /(100 \sqrt{12}))$ is treated. In this situation there is a finite resistance to bending which tends to stiffen the structure, increasing the natural frequencies. As noted above, the predictions of Niordson - Eqs. (18 19) - differ from those by Junger \& Feit - Eqs. (21,22) because the effect of bending stiffness is taken into account differently. However, it is observed that neither prediction is fully consistent with the value obtained through accurate numerical simulations. The relative errors $\epsilon=\left|\omega_{0}-\widehat{\omega}_{0}\right| / \widehat{\omega}_{0}$ are plotted versus the characteristic element dimension $h$ in Fig. $13 \mathrm{~b}$. The value $\widehat{\omega}_{0}=3.741698147 \times 10^{3} \mathrm{~s}^{-1}$, obtained using a highly refined mesh with deltoidal icositetrahedral topology, was used to calculate $\epsilon$. Notice in Fig. $13 \mathrm{~b}$ that all conforming mesh topologies yield convergence to the same numerical result. Both Niordson's and Junger \& Feit's predictions are consistent with our numerical reference value up to a relative difference around $10^{-5}$. The discrepancy between the theoretical predictions is of the same order of magnitude. Because the theoretical results disagree with each other while the numerical approximation is the same for all topologies, we presume that the latter is more reliable at higher precisions. In any case, the value $\omega_{0}=3.7417 \times 10^{3} \mathrm{~s}^{-1}$ can be used as a safe estimate. A summary of these results is shown in Table 1 .

\section{Conclusion}

In this work, the composite mapping method of Cobb is applied to find Bézier patches that tile the unit sphere. A general method for generating a convex, quadrangular spherical tile is described. We provide a MATLAB implementation of the algorithm in the supplementary material to this manuscript. Using this method, five novel degeneracy-free Bézier tessellations of the sphere are obtained. The topologies of these tilings are derived from the icosahedron and from four of the Catalan solids: the rhombic dodecahedron, the rhombic triacontahedron, the deltoidal icositetrahedron and the deltoidal hexecontahedron. The first tessellation

Table 1: Estimates of the lowest nonzero pulsation $\omega_{0}$ of a vibrating sphere. $R=1 \mathrm{~m}, t=0.01$ $\mathrm{m}, E=70 \mathrm{GPa}, \nu=0.3$ and $\rho=2700 \mathrm{~kg} \mathrm{~m}^{-3}$

\begin{tabular}{ll}
\hline & $\omega_{0}\left[\mathrm{~s}^{-1}\right]$ \\
\hline Niordson [57] & $3.74174947 \times 10^{3}$ \\
Junger \& Feit [42] & $3.74170822 \times 10^{3}$ \\
Numerical reference & $3.74169815 \times 10^{3}$ \\
\hline
\end{tabular}




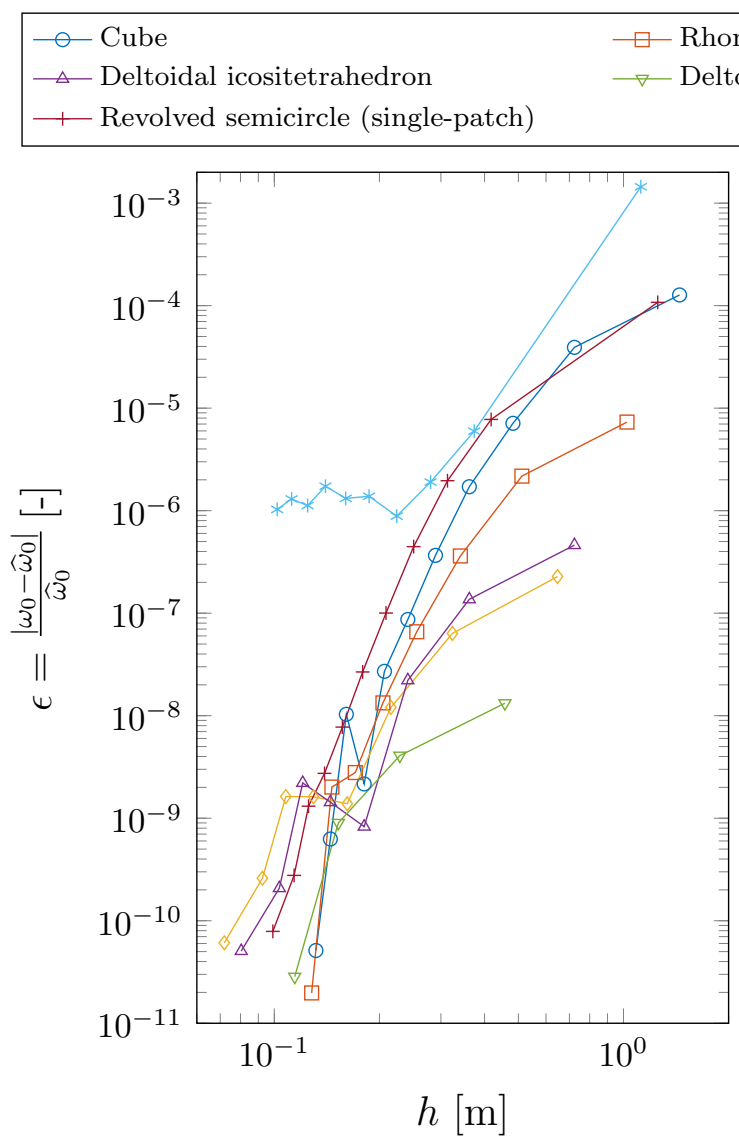

(a)

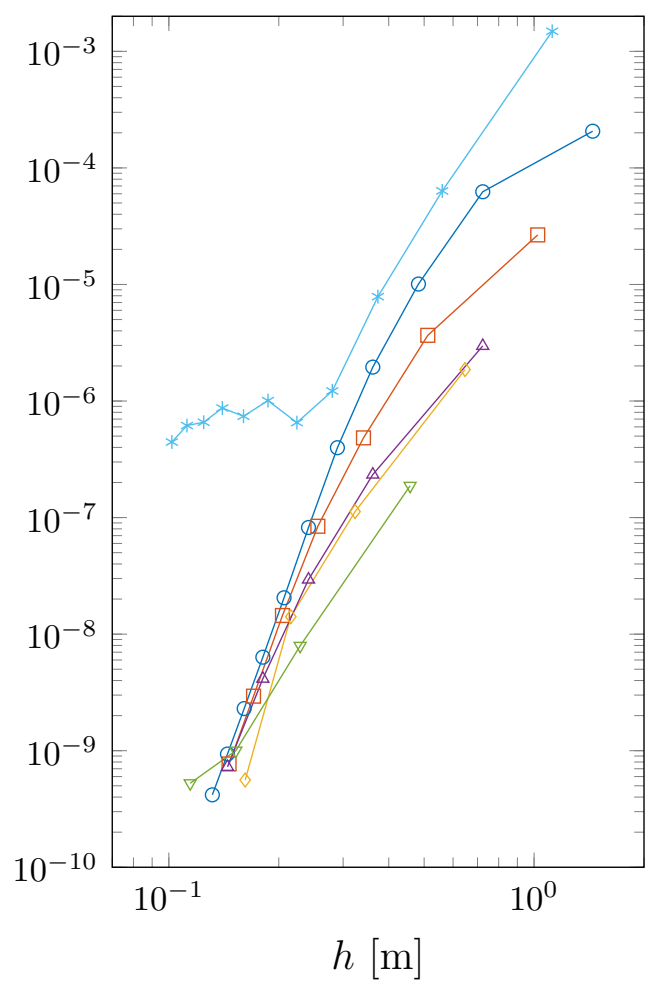

(b)

Figure 13: Convergence of $\omega_{0}$ for meshes of different topologies. (a) Excluding the effect of bending stiffness (spherical membrane). Errors are computed with respect to $\widehat{\omega}_{0}=$ $3.7413348277488 \times 10^{3} \mathrm{~s}^{-1}$. This is an analytic solution [42, 57]. (b) Including the effect of bending stiffness. Errors are computed with respect to $\widehat{\omega}_{0}=3.741698147 \times 10^{3} \mathrm{~s}^{-1}$. This is a numerical approximation obtained on a fine grid. The single-patch discretization is not employed here since it already has poor performance when only $C^{0}$-continuity is imposed. 
consists of 10 patches and has an intrinsically nonconforming mesh. The others have conforming meshes and consist of respectively 12, 30, 24 and 60 patches. The geometric data of these tilings are available as supplementary material. We then proceed to apply the tilings in three numerical case studies, proposed as benchmark tests for multipatch isogeometric methods. An acoustic scattering problem is studied to investigate $C^{0}$-coupling. For testing $C^{1}$-coupling, two problems involving Kirchhoff-Love shells are proposed. In these tests, good convergence is obtained using all meshes except the intrinsically nonconforming one. This mesh presents a particularly severe test for multipatch isogeometric methods, as the nonconformity is not the result of unequal knot vectors.

\section{Acknowledgements}

This work was supported by the Research Fund KU Leuven, and by Flanders Make, the strategic research centre for the manufacturing industry. The research

of F. Maurin is funded by an Experienced Researcher grant within the European ANTARES Project, under the FP7 Marie Curie Programme (GA606817). 


\title{
Appendix A. Control point data
}

\author{
Appendix A.1. Cube (Fig. 5a)
}

Table A.2: Control net for a tile of the cube topology. Expressions in terms of radicals can be found in [5].

\begin{tabular}{lllll}
\hline Index & $x$ & $y$ & $z$ & $w$ \\
\hline$(1,1)$ & 0.577350269189626 & 0.577350269189626 & -0.577350269189626 & 1 \\
$(1,2)$ & 0.278838767912603 & 0.632392158505876 & -0.632392158505876 & 0.891211203608397 \\
$(1,3)$ & 0 & 0.647791890991355 & -0.647791890991355 & 0.859116756396542 \\
$(1,4)$ & -0.278838767912603 & 0.632392158505876 & -0.632392158505876 & 0.891211203608397 \\
$(1,5)$ & -0.577350269189626 & 0.577350269189626 & -0.577350269189626 & 1 \\
& & & & \\
$(2,1)$ & 0.632392158505876 & 0.278838767912603 & -0.632392158505876 & 0.891211203608397 \\
$(2,2)$ & 0.315090742770461 & 0.315090742770461 & -0.762259526419164 & 0.762259526419164 \\
$(2,3)$ & 0 & 0.328648516366383 & -0.804938188574224 & 0.71866517354005 \\
$(2,4)$ & -0.315090742770461 & 0.315090742770461 & -0.762259526419164 & 0.762259526419164 \\
$(2,5)$ & -0.632392158505876 & 0.278838767912603 & -0.632392158505876 & 0.891211203608397 \\
& & & & \\
$(3,1)$ & 0.647791890991355 & 0 & -0.647791890991355 & 0.859116756396542 \\
$(3,2)$ & 0.328648516366383 & 0 & 0.804938188574224 & 0.71866517354005 \\
$(3,3)$ & 0 & 0 & -0.859116756396542 & 0.671272431591931 \\
$(3,4)$ & -0.328648516366383 & 0 & -0.804938188574224 & 0.71866517354005 \\
$(3,5)$ & -0.647791890991355 & 0 & -0.647791890991355 & 0.859116756396542 \\
& & & & \\
$(4,1)$ & 0.632392158505876 & -0.278838767912603 & -0.632392158505876 & 0.891211203608397 \\
$(4,2)$ & 0.315090742770461 & -0.315090742770461 & -0.762259526419164 & 0.762259526419164 \\
$(4,3)$ & 0 & -0.328648516366383 & -0.804938188574224 & 0.71866517354005 \\
$(4,4)$ & -0.315090742770461 & -0.315090742770461 & -0.762259526419164 & 0.762259526419164 \\
$(4,5)$ & -0.632392158505876 & -0.278838767912603 & -0.632392158505876 & 0.891211203608397 \\
& & & & \\
$(5,1)$ & 0.577350269189626 & -0.577350269189626 & -0.577350269189626 & 1 \\
$(5,2)$ & 0.278838767912603 & -0.632392158505876 & -0.632392158505876 & 0.891211203608397 \\
$(5,3)$ & 0 & -0.647791890991355 & -0.647791890991355 & 0.859116756396542 \\
$(5,4)$ & -0.278838767912603 & -0.632392158505876 & -0.632392158505876 & 0.891211203608397 \\
$(5,5)$ & -0.577350269189626 & -0.577350269189626 & -0.577350269189626 & 1 \\
& & & &
\end{tabular}


Table A.3: Rotations for constructing the cube. The control net for tile 1 is given in Table A.2. See also [5]

\begin{tabular}{llcc}
\hline Tile & Parent & Rotation axis & Angle \\
\hline 1 & - & - & - \\
2 & 1 & $(0,1,0)$ & $-\pi / 2$ \\
& & & \\
3 & 2 & $(0,0,1)$ & $-\pi / 2$ \\
4 & 3 & $(0,0,1)$ & $-\pi / 2$ \\
5 & 4 & $(0,0,1)$ & $-\pi / 2$ \\
6 & 1 & $(0,1,0)$ & $\pi$
\end{tabular}


Appendix A.2. Paired Icosahedron (Fig. 5b)

Table A.4: Control net for a tile of the paired icosahedral topology.

\begin{tabular}{lllll}
\hline Index & $x$ & $y$ & $z$ & $w$ \\
\hline$(1,1)$ & 0.525731112119134 & 0 & -0.85065080835204 & 1 \\
$(1,2)$ & 0.429796348885117 & 0.275336158073158 & -0.865592204757753 & 0.962274021164523 \\
$(1,3)$ & 0.311717938145732 & 0.534562651383477 & -0.834748106494081 & 0.985710269297313 \\
$(1,4)$ & 0.170167104021026 & 0.784887241579794 & -0.760423150705621 & 1.06744307521666 \\
$(1,5)$ & 0 & 1.03180540371592 & -0.637690809272245 & 1.2129599990798 \\
& & & & \\
$(2,1)$ & 0.429796348885117 & -0.275336158073158 & -0.865592204757753 & 0.962274021164523 \\
$(2,2)$ & 0.29950484262419 & 0 & -0.939320366320159 & 0.888686117611397 \\
$(2,3)$ & 0.156716276824699 & 0.268482619299878 & -0.947574924704289 & 0.880291301217988 \\
$(2,4)$ & 0 & 0.529897664002098 & -0.888686117611397 & 0.939320366320159 \\
$(2,5)$ & -0.170167104021026 & 0.784887241579794 & -0.760423150705621 & 1.06744307521666 \\
& & & & \\
$(3,1)$ & 0.311717938145732 & -0.534562651383477 & -0.834748106494081 & 0.985710269297313 \\
$(3,2)$ & 0.156716276824699 & -0.268482619299878 & -0.947574924704289 & 0.880291301217988 \\
$(3,3)$ & 0 & 0 & -0.985710269297313 & 0.844812250680963 \\
$(3,4)$ & -0.156716276824699 & 0.268482619299878 & -0.947574924704289 & 0.880291301217988 \\
$(3,5)$ & -0.311717938145732 & 0.534562651383477 & -0.834748106494081 & 0.985710269297313 \\
& & & & \\
$(4,1)$ & 0.170167104021026 & -0.784887241579794 & -0.760423150705621 & 1.06744307521666 \\
$(4,2)$ & 0 & -0.529897664002098 & -0.888686117611397 & 0.939320366320159 \\
$(4,3)$ & -0.156716276824699 & -0.268482619299878 & -0.947574924704289 & 0.880291301217988 \\
$(4,4)$ & -0.29950484262419 & 0 & -0.939320366320159 & 0.888686117611397 \\
$(4,5)$ & -0.429796348885117 & 0.275336158073158 & -0.865592204757753 & 0.962274021164523 \\
& & & & \\
$(5,1)$ & 0 & -1.03180540371592 & -0.637690809272245 & 1.2129599990798 \\
$(5,2)$ & -0.170167104021026 & -0.784887241579794 & -0.760423150705621 & 1.06744307521666 \\
$(5,3)$ & -0.311717938145732 & -0.534562651383477 & -0.834748106494081 & 0.985710269297313 \\
$(5,4)$ & -0.429796348885117 & -0.275336158073158 & -0.865592204757753 & 0.962274021164523 \\
$(5,5)$ & -0.525731112119134 & 0 & -0.85065080835204 & 1 \\
& & & &
\end{tabular}


Table A.5: Rotations for constructing the spherical paired icosahedron. The control net for tile 1 is given in Table A.4. In this table, $C_{0}=\frac{1+\sqrt{5}}{2}$.

\begin{tabular}{llcc}
\hline Tile & Parent & Rotation axis & Angle \\
\hline 1 & - & - & - \\
2 & 1 & $\left(0, C_{0},-1\right)$ & $2 \pi / 5$ \\
3 & 2 & $\left(0, C_{0},-1\right)$ & $2 \pi / 5$ \\
4 & 3 & $\left(0, C_{0},-1\right)$ & $2 \pi / 5$ \\
5 & 4 & $\left(0, C_{0},-1\right)$ & $2 \pi / 5$ \\
& & & \\
6 & 1 & $(1,0,0)$ & $\pi$ \\
& & & \\
7 & 6 & $\left(0, C_{0},-1\right)$ & $2 \pi / 5$ \\
8 & 7 & $\left(0, C_{0},-1\right)$ & $2 \pi / 5$ \\
9 & 8 & $\left(0, C_{0},-1\right)$ & $2 \pi / 5$ \\
10 & 9 & $\left(0, C_{0},-1\right)$ & $2 \pi / 5$
\end{tabular}


Appendix A.3. Rhombic Dodecahedron (Fig. 5c)

Table A.6: Control net for a tile of the rhombic dodecahedral topology.

\begin{tabular}{lllll}
\hline Index & $x$ & $y$ & $z$ & $w$ \\
\hline$(1,1)$ & 0.707106781186548 & 0 & -0.707106781186548 & 1 \\
$(1,2)$ & 0.543498332898695 & 0.157559051751283 & -0.766320480760004 & 0.926181765263785 \\
$(1,3)$ & 0.372942808261694 & 0.298711835726882 & -0.795385137588014 & 0.892332225930114 \\
$(1,4)$ & 0.192969640573706 & 0.42652917743322 & -0.796172988047606 & 0.896329257976183 \\
$(1,5)$ & 0 & 0.542582116587371 & -0.76732698797896 & 0.939779793207588 \\
& & & & \\
$(2,1)$ & 0.543498332898695 & -0.157559051751283 & -0.766320480760004 & 0.926181765263785 \\
$(2,2)$ & 0.368095453844589 & 0 & -0.839011327341959 & 0.853553390593274 \\
$(2,3)$ & 0.187067875769969 & 0.149867722114512 & -0.867919252547924 & 0.824582993475865 \\
$(2,4)$ & 0 & 0.291856641368583 & -0.853553390593274 & 0.839011327341959 \\
$(2,5)$ & -0.192969640573706 & 0.42652917743322 & -0.796172988047606 & 0.896329257976183 \\
& & & & \\
$(3,1)$ & 0.372942808261694 & -0.298711835726882 & -0.795385137588014 & 0.892332225930114 \\
$(3,2)$ & 0.187067875769969 & -0.149867722114512 & -0.867919252547924 & 0.824582993475865 \\
$(3,3)$ & 0 & 0 & -0.892332225930114 & 0.801848276810821 \\
$(3,4)$ & -0.187067875769969 & 0.149867722114512 & -0.867919252547924 & 0.824582993475865 \\
$(3,5)$ & -0.372942808261694 & 0.298711835726882 & -0.795385137588014 & 0.892332225930114 \\
& & & & \\
$(4,1)$ & 0.192969640573706 & -0.42652917743322 & -0.796172988047606 & 0.896329257976183 \\
$(4,2)$ & 0 & -0.291856641368583 & -0.853553390593274 & 0.839011327341959 \\
$(4,3)$ & -0.187067875769969 & -0.149867722114512 & -0.867919252547924 & 0.824582993475865 \\
$(4,4)$ & -0.368095453844589 & 0 & -0.839011327341959 & 0.853553390593274 \\
$(4,5)$ & -0.543498332898695 & 0.157559051751283 & -0.766320480760004 & 0.926181765263785 \\
& & & & \\
$(5,1)$ & 0 & -0.542582116587371 & -0.76732698797896 & 0.939779793207588 \\
$(5,2)$ & -0.192969640573706 & -0.42652917743322 & -0.796172988047606 & 0.896329257976183 \\
$(5,3)$ & -0.372942808261694 & -0.298711835726882 & -0.795385137588014 & 0.892332225930114 \\
$(5,4)$ & -0.543498332898695 & -0.157559051751283 & -0.766320480760004 & 0.926181765263785 \\
$(5,5)$ & -0.707106781186548 & 0 & -0.707106781186548 & 1 \\
& & & &
\end{tabular}


Table A.7: Rotations for constructing the spherical rhombic dodecahedron. The control net for tile 1 is given in Table A.6. In this table, $C_{1}=\frac{\sqrt{2}}{2}$.

\begin{tabular}{llcc}
\hline Tile & Parent & Rotation axis & Angle \\
\hline 1 & - & - & - \\
2 & 1 & $\left(C_{1}, 0,-C_{1}\right)$ & $\pi / 2$ \\
3 & 2 & $\left(C_{1}, 0,-C_{1}\right)$ & $\pi / 2$ \\
4 & 3 & $\left(C_{1}, 0,-C_{1}\right)$ & $\pi / 2$ \\
& & & \\
5 & 1 & $\left(-C_{1}, 0,-C_{1}\right)$ & $\pi / 2$ \\
6 & 5 & $\left(-C_{1}, 0,-C_{1}\right)$ & $\pi / 2$ \\
7 & 6 & $\left(-C_{1}, 0,-C_{1}\right)$ & $\pi / 2$ \\
& & $(1,0,0)$ & $\pi$ \\
8 & 1 & $(1,0,0)$ & $\pi$ \\
9 & 2 & $(1,0,0)$ & $\pi$ \\
10 & 4 & $(1,0,0)$ & $\pi$ \\
11 & 5 & $(1,0,0)$ & $\pi$
\end{tabular}


Appendix A.4. Rhombic Triacontahedron (Fig. 5d)

Table A.8: Control net for a tile of the rhombic triacontahedral topology.

\begin{tabular}{lllll}
\hline Index & $x$ & $y$ & $z$ & $w$ \\
\hline$(1,1)$ & 0.525731112119136 & 0 & -0.85065080835204 & 1 \\
$(1,2)$ & 0.39822501603901 & 0.0921151302387952 & -0.88550215296488 & 0.962612402772894 \\
$(1,3)$ & 0.268333463946774 & 0.179554148904023 & -0.904251529636665 & 0.943019925793728 \\
$(1,4)$ & 0.135719708088242 & 0.262588070476675 & -0.907063594178177 & 0.941050961559596 \\
$(1,5)$ & 0 & 0.341413776190337 & -0.893832870293752 & 0.956817938058288 \\
& & & & \\
$(2,1)$ & 0.39822501603901 & -0.0921151302387952 & -0.88550215296488 & 0.962612402772894 \\
$(2,2)$ & 0.266966486974972 & 0 & -0.91868995856938 & 0.929426335091425 \\
$(2,3)$ & 0.134230690050623 & 0.0898222435321767 & -0.933315412634673 & 0.914799143103101 \\
$(2,4)$ & 0 & 0.177342333701809 & -0.929426335091425 & 0.91868995856938 \\
$(2,5)$ & -0.135719708088242 & 0.262588070476675 & -0.907063594178177 & 0.941050961559596 \\
& & & & \\
$(3,1)$ & 0.268333463946774 & -0.179554148904023 & -0.904251529636665 & 0.943019925793728 \\
$(3,2)$ & 0.134230690050623 & -0.0898222435321767 & -0.933315412634673 & 0.914799143103101 \\
$(3,3)$ & 0 & 0 & -0.943019925793728 & 0.905377980610547 \\
$(3,4)$ & -0.134230690050623 & 0.0898222435321767 & -0.933315412634673 & 0.914799143103101 \\
$(3,5)$ & -0.268333463946774 & 0.179554148904023 & -0.904251529636665 & 0.943019925793728 \\
& & & & \\
$(4,1)$ & 0.135719708088242 & -0.262588070476675 & -0.907063594178177 & 0.941050961559596 \\
$(4,2)$ & 0 & -0.177342333701809 & -0.929426335091425 & 0.91868995856938 \\
$(4,3)$ & -0.134230690050623 & -0.0898222435321767 & -0.933315412634673 & 0.914799143103101 \\
$(4,4)$ & -0.266966486974972 & 0 & -0.91868995856938 & 0.929426335091425 \\
$(4,5)$ & -0.39822501603901 & 0.0921151302387952 & -0.88550215296488 & 0.962612402772894 \\
& & & & \\
$(5,1)$ & 0 & -0.341413776190337 & -0.893832870293752 & 0.956817938058288 \\
$(5,2)$ & -0.135719708088242 & -0.262588070476675 & -0.907063594178177 & 0.941050961559596 \\
$(5,3)$ & -0.268333463946774 & -0.179554148904023 & -0.904251529636665 & 0.943019925793728 \\
$(5,4)$ & -0.39822501603901 & -0.0921151302387952 & -0.88550215296488 & 0.962612402772894 \\
$(5,5)$ & -0.525731112119136 & 0 & -0.85065080835204 & 1 \\
& & & &
\end{tabular}


Table A.9: Rotations for constructing the spherical rhombic triacontahedron. The control net for tile 1 is given in Table A.8. In this table, $C_{2}=\left(\frac{1+\sqrt{5}}{2}\right)$.

\begin{tabular}{|c|c|c|c|}
\hline Tile & Parent & Rotation axis & Angle \\
\hline 1 & - & - & - \\
\hline 2 & 1 & $\left(C_{2}^{2}, 0,-C_{2}^{3}\right)$ & $2 \pi / 5$ \\
\hline 3 & 2 & $\left(C_{2}^{2}, 0,-C_{2}^{3}\right)$ & $2 \pi / 5$ \\
\hline 4 & 3 & $\left(C_{2}^{2}, 0,-C_{2}^{3}\right)$ & $2 \pi / 5$ \\
\hline 5 & 1 & $\left(-C_{2}^{2}, 0,-C_{2}^{3}\right)$ & $2 \pi / 5$ \\
\hline 6 & 2 & $\left(-C_{2}^{2}, 0,-C_{2}^{3}\right)$ & $2 \pi / 5$ \\
\hline 7 & 3 & $\left(-C_{2}^{2}, 0,-C_{2}^{3}\right)$ & $2 \pi / 5$ \\
\hline 8 & 4 & $\left(-C_{2}^{2}, 0,-C_{2}^{3}\right)$ & $2 \pi / 5$ \\
\hline 9 & 5 & $\left(-C_{2}^{2}, 0,-C_{2}^{3}\right)$ & $2 \pi / 5$ \\
\hline 10 & 6 & $\left(-C_{2}^{2}, 0,-C_{2}^{3}\right)$ & $2 \pi / 5$ \\
\hline 11 & 7 & $\left(-C_{2}^{2}, 0,-C_{2}^{3}\right)$ & $2 \pi / 5$ \\
\hline 12 & 8 & $\left(-C_{2}^{2}, 0,-C_{2}^{3}\right)$ & $2 \pi / 5$ \\
\hline 13 & 9 & $\left(-C_{2}^{2}, 0,-C_{2}^{3}\right)$ & $2 \pi / 5$ \\
\hline 14 & 10 & $\left(-C_{2}^{2}, 0,-C_{2}^{3}\right)$ & $2 \pi / 5$ \\
\hline 15 & 11 & $\left(-C_{2}^{2}, 0,-C_{2}^{3}\right)$ & $2 \pi / 5$ \\
\hline 16 & 12 & $\left(-C_{2}^{2}, 0,-C_{2}^{3}\right)$ & $2 \pi / 5$ \\
\hline 17 & 13 & $\left(-C_{2}^{2}, 0,-C_{2}^{3}\right)$ & $2 \pi / 5$ \\
\hline 18 & 14 & $\left(-C_{2}^{2}, 0,-C_{2}^{3}\right)$ & $2 \pi / 5$ \\
\hline 19 & 15 & $\left(-C_{2}^{2}, 0,-C_{2}^{3}\right)$ & $2 \pi / 5$ \\
\hline 20 & 16 & $\left(-C_{2}^{2}, 0,-C_{2}^{3}\right)$ & $2 \pi / 5$ \\
\hline 21 & 1 & $(0,1,0)$ & $\pi$ \\
\hline 22 & 5 & $(0,1,0)$ & $\pi$ \\
\hline 23 & 9 & $(0,1,0)$ & $\pi$ \\
\hline 24 & 13 & $(0,1,0)$ & $\pi$ \\
\hline 25 & 17 & $(0,1,0)$ & $\pi$ \\
\hline 26 & 2 & $(0,1,0)$ & $\pi$ \\
\hline 27 & 6 & $(0,1,0)$ & $\pi$ \\
\hline 28 & 10 & $(0,1,0)$ & $\pi$ \\
\hline 29 & 14 & $(0,1,0)$ & $\pi$ \\
\hline 30 & 18 & $(0,1,0)$ & $\pi$ \\
\hline
\end{tabular}


Appendix A.5. Deltoidal icositetrahedron (Fig. 5e)

Table A.10: Control net for a tile of the deltoidal icositetrahedral topology.

\begin{tabular}{lllll}
\hline Index & $x$ & $y$ & $z$ & $w$ \\
\hline$(1,1)$ & 0 & 0 & -1 & 1 \\
$(1,2)$ & 0.207106781186548 & 0 & -1 & 1 \\
$(1,3)$ & 0.414213562373095 & 0 & -0.971404520791032 & 1.02859547920897 \\
$(1,4)$ & 0.621320343559643 & 0 & -0.914213562373095 & 1.0857864376269 \\
$(1,5)$ & 0.82842712474619 & 0 & -0.82842712474619 & 1.17157287525381 \\
& & & & \\
$(2,1)$ & 0 & 0.207106781186548 & -1 & 1 \\
$(2,2)$ & 0.20246636350282 & 0.20246636350282 & -0.998930607671726 & 0.998930607671726 \\
$(2,3)$ & 0.407140425183055 & 0.197167079290905 & -0.969834223875419 & 1.02446235037379 \\
$(2,4)$ & 0.613579228234834 & 0.191208928550803 & -0.911155682104475 & 1.07815039461279 \\
$(2,5)$ & 0.821339815852291 & 0.184591911282515 & -0.821339815852291 & 1.16154990689533 \\
& & & & \\
$(3,1)$ & 0 & 0.414213562373095 & -0.971404520791032 & 1.02859547920897 \\
$(3,2)$ & 0.197167079290905 & 0.407140425183055 & -0.969834223875419 & 1.02446235037379 \\
$(3,3)$ & 0.396726193243811 & 0.396726193243811 & -0.942520631201463 & 1.04607658343011 \\
$(3,4)$ & 0.598682394601221 & 0.38300296952756 & -0.887573768782017 & 1.09534035076458 \\
$(3,5)$ & 0.802955081964821 & 0.366025403784439 & -0.802955081964822 & 1.17432880222789 \\
& & & & \\
$(4,1)$ & 0 & 0.621320343559643 & -0.914213562373095 & 1.0857864376269 \\
$(4,2)$ & 0.191208928550803 & 0.613579228234834 & -0.911155682104475 & 1.07815039461279 \\
$(4,3)$ & 0.38300296952756 & 0.598682394601221 & -0.887573768782017 & 1.09534035076458 \\
$(4,4)$ & 0.576618885956231 & 0.576618885956231 & -0.84219639933622 & 1.13862772915177 \\
$(4,5)$ & 0.773563913640501 & 0.547485916996871 & -0.773563913640501 & 1.20932580910712 \\
& & & & \\
$(5,1)$ & 0 & 0.82842712474619 & -0.82842712474619 & 1.17157287525381 \\
$(5,2)$ & 0.184591911282515 & 0.821339815852291 & -0.821339815852291 & 1.16154990689533 \\
$(5,3)$ & 0.366025403784439 & 0.802955081964821 & -0.802955081964822 & 1.17432880222789 \\
$(5,4)$ & 0.547485916996871 & 0.773563913640501 & -0.773563913640501 & 1.20932580910712 \\
$(5,5)$ & 0.732050807568877 & 0.732050807568877 & -0.732050807568877 & 1.26794919243112
\end{tabular}


Table A.11: Rotations for constructing the spherical deltoidal icositetrahedron. The control net for tile 1 is given in Table A.10.

\begin{tabular}{llcc}
\hline Tile & Parent & Rotation axis & Angle \\
\hline 1 & - & - & - \\
2 & 1 & $(0,0,1)$ & $\pi / 2$ \\
3 & 2 & $(0,0,1)$ & $\pi / 2$ \\
4 & 3 & $(0,0,1)$ & $\pi / 2$ \\
& & & \\
5 & 1 & $(1,0,0)$ & $\pi / 2$ \\
6 & 2 & $(1,0,0)$ & $\pi / 2$ \\
7 & 3 & $(1,0,0)$ & $\pi / 2$ \\
8 & 4 & $(1,0,0)$ & $\pi / 2$ \\
9 & 5 & $(1,0,0)$ & $\pi / 2$ \\
10 & 6 & $(1,0,0)$ & $\pi / 2$ \\
11 & 7 & $(1,0,0)$ & $\pi / 2$ \\
12 & 8 & $(1,0,0)$ & $\pi / 2$ \\
13 & 9 & $(1,0,0)$ & $\pi / 2$ \\
14 & 10 & $(1,0,0)$ & $\pi / 2$ \\
15 & 11 & $(1,0,0)$ & $\pi / 2$ \\
16 & 12 & $(1,0,0)$ & $\pi / 2$ \\
& & & \\
17 & 1 & $(0,1,0)$ & $\pi / 2$ \\
18 & 2 & $(0,1,0)$ & $\pi / 2$ \\
19 & 3 & $(0,1,0)$ & $\pi / 2$ \\
20 & 4 & $(0,1,0)$ & $\pi / 2$ \\
& & $(0,1,0)$ & $-\pi / 2$ \\
21 & 1 & $(0,1,0)$ & $-\pi / 2$ \\
22 & 2 & $(0,1,0)$ & $-\pi / 2$ \\
23 & 3 & $(0,1,0)$ & $-\pi / 2$ \\
24 & 4 & &
\end{tabular}


Appendix A.6. Deltoidal hexecontahedron (Fig. 5f)

Table A.12: Control net for a tile of the deltoidal hexecontahedral topology.

\begin{tabular}{|c|c|c|c|c|}
\hline Index & $x$ & $y$ & $z$ & $w$ \\
\hline$(1,1)$ & 0.525731112119134 & 0 & -0.85065080835204 & 1 \\
\hline$(1,2)$ & 0.41744773371802 & 0.0782943772341414 & -0.880421962409361 & 0.968396915313599 \\
\hline$(1,3)$ & 0.307403110285292 & 0.153237961093481 & -0.898570871198503 & 0.949638571914636 \\
\hline$(1,4)$ & 0.195389549716671 & 0.224995400187641 & -0.90519253749174 & 0.94362634023122 \\
\hline$(1,5)$ & 0.0811746845804661 & 0.293692767838179 & -0.90024104712763 & 0.95040976122441 \\
\hline$(2,1)$ & 0.417447733718021 & -0.0782943772341413 & -0.880421962409361 & 0.968396915313599 \\
\hline$(2,2)$ & 0.323693127933988 & 0 & -0.903965913805063 & 0.944617730618712 \\
\hline$(2,3)$ & 0.229140845494387 & 0.0758774152168262 & -0.917097970830361 & 0.93170215543976 \\
\hline$(2,4)$ & 0.133736708743163 & 0.149394055669103 & -0.920228967255028 & 0.929241169406929 \\
\hline$(2,5)$ & 0.0374322338447202 & 0.220602104543352 & -0.9137296130987 & 0.936864005889569 \\
\hline$(3,1)$ & 0.307403110285292 & -0.153237961093482 & -0.898570871198503 & 0.949638571914636 \\
\hline$(3,2)$ & 0.229140845494387 & -0.0758774152168271 & -0.917097970830362 & 0.931702155439761 \\
\hline$(3,3)$ & 0.150824464864122 & 0 & -0.926904886553933 & 0.922486587196324 \\
\hline$(3,4)$ & 0.0724659822096831 & 0.0743902842177182 & -0.92841206298977 & 0.921570922185131 \\
\hline$(3,5)$ & -0.00592281916176617 & 0.147292766844402 & -0.92202912788654 & 0.928545429158656 \\
\hline$(4,1)$ & 0.195389549716671 & -0.224995400187641 & -0.90519253749174 & 0.94362634023122 \\
\hline$(4,2)$ & 0.13373670874316 & -0.149394055669105 & -0.92022896725503 & 0.929241169406929 \\
\hline$(4,3)$ & 0.0724659822096804 & -0.0743902842177203 & -0.928412062989769 & 0.921570922185131 \\
\hline$(4,4)$ & 0.0115864762918693 & 0 & -0.929972065922228 & 0.920385413950595 \\
\hline$(4,5)$ & -0.0488932135158582 & 0.0737602585150586 & -0.925140907875166 & 0.925452711113102 \\
\hline$(5,1)$ & 0.0811746845804662 & -0.293692767838179 & -0.90024104712763 & 0.95040976122441 \\
\hline$(5,2)$ & 0.0374322338447153 & -0.220602104543355 & -0.913729613098698 & 0.936864005889567 \\
\hline$(5,3)$ & -0.00592281916177271 & -0.147292766844406 & -0.922029127886541 & 0.928545429158658 \\
\hline$(5,4)$ & -0.0488932135158631 & -0.0737602585150609 & -0.925140907875165 & 0.925452711113101 \\
\hline$(5,5)$ & -0.0914815456150609 & 0 & -0.923064341972834 & 0.927586466379206 \\
\hline
\end{tabular}


Table A.13: Rotations for constructing the spherical deltoidal hexecontahedron. The sphere consists of tiles 1 to 60 . The control net for tile 0 is given in Table A.12. In this table, $C_{3}=\frac{1+\sqrt{5}}{2}$, $C_{4}=\frac{1+\sqrt{5}}{6}$ and $C_{5}=\frac{2+\sqrt{5}}{3}$.

\begin{tabular}{|c|c|c|c|c|c|c|c|}
\hline Tile & Parent & Axis of rotation & Angle of rotation & Tile & Parent & Axis of rotation & Angle of rotation \\
\hline \multirow[t]{2}{*}{1} & 0 & $\left(1,0,-C_{3}\right)$ & $\pi / 5$ & 31 & 1 & $(1,0,0)$ & $\pi$ \\
\hline & & & & 32 & 2 & $(1,0,0)$ & $\pi$ \\
\hline 2 & 1 & $\left(0, C_{4},-C_{5}\right)$ & $2 \pi / 3$ & 33 & 3 & $(1,0,0)$ & $\pi$ \\
\hline \multirow[t]{2}{*}{3} & 2 & $\left(0, C_{4},-C_{5}\right)$ & $2 \pi / 3$ & 34 & 4 & $(1,0,0)$ & $\pi$ \\
\hline & & & & 35 & 5 & $(1,0,0)$ & $\pi$ \\
\hline 4 & 1 & $\left(1,0,-C_{3}\right)$ & $-2 \pi / 5$ & 36 & 6 & $(1,0,0)$ & $\pi$ \\
\hline 5 & 2 & $\left(1,0,-C_{3}\right)$ & $-2 \pi / 5$ & 37 & 7 & $(1,0,0)$ & $\pi$ \\
\hline \multirow[t]{2}{*}{6} & 3 & $\left(1,0,-C_{3}\right)$ & $-2 \pi / 5$ & 38 & 8 & $(1,0,0)$ & $\pi$ \\
\hline & & & & 39 & 9 & $(1,0,0)$ & $\pi$ \\
\hline 7 & 1 & $\left(0, C_{3},-1\right)$ & $2 \pi / 5$ & 40 & 10 & $(1,0,0)$ & $\pi$ \\
\hline 8 & 2 & $\left(0, C_{3},-1\right)$ & $2 \pi / 5$ & 41 & 11 & $(1,0,0)$ & $\pi$ \\
\hline 9 & 3 & $\left(0, C_{3},-1\right)$ & $2 \pi / 5$ & 42 & 12 & $(1,0,0)$ & $\pi$ \\
\hline 10 & 4 & $\left(0, C_{3},-1\right)$ & $2 \pi / 5$ & 43 & 13 & $(1,0,0)$ & $\pi$ \\
\hline 11 & 5 & $\left(0, C_{3},-1\right)$ & $2 \pi / 5$ & 44 & 14 & $(1,0,0)$ & $\pi$ \\
\hline 12 & 6 & $\left(0, C_{3},-1\right)$ & $2 \pi / 5$ & 45 & 15 & $(1,0,0)$ & $\pi$ \\
\hline 13 & 7 & $\left(0, C_{3},-1\right)$ & $2 \pi / 5$ & 46 & 16 & $(1,0,0)$ & $\pi$ \\
\hline 14 & 8 & $\left(0, C_{3},-1\right)$ & $2 \pi / 5$ & 47 & 17 & $(1,0,0)$ & $\pi$ \\
\hline 15 & 9 & $\left(0, C_{3},-1\right)$ & $2 \pi / 5$ & 48 & 18 & $(1,0,0)$ & $\pi$ \\
\hline 16 & 10 & $\left(0, C_{3},-1\right)$ & $2 \pi / 5$ & 49 & 19 & $(1,0,0)$ & $\pi$ \\
\hline 17 & 11 & $\left(0, C_{3},-1\right)$ & $2 \pi / 5$ & 50 & 20 & $(1,0,0)$ & $\pi$ \\
\hline 18 & 12 & $\left(0, C_{3},-1\right)$ & $2 \pi / 5$ & 51 & 21 & $(1,0,0)$ & $\pi$ \\
\hline 19 & 13 & $\left(0, C_{3},-1\right)$ & $2 \pi / 5$ & 52 & 22 & $(1,0,0)$ & $\pi$ \\
\hline 20 & 14 & $\left(0, C_{3},-1\right)$ & $2 \pi / 5$ & 53 & 23 & $(1,0,0)$ & $\pi$ \\
\hline 21 & 15 & $\left(0, C_{3},-1\right)$ & $2 \pi / 5$ & 54 & 24 & $(1,0,0)$ & $\pi$ \\
\hline 22 & 16 & $\left(0, C_{3},-1\right)$ & $2 \pi / 5$ & 55 & 25 & $(1,0,0)$ & $\pi$ \\
\hline 23 & 17 & $\left(0, C_{3},-1\right)$ & $2 \pi / 5$ & 56 & 26 & $(1,0,0)$ & $\pi$ \\
\hline 24 & 18 & $\left(0, C_{3},-1\right)$ & $2 \pi / 5$ & 57 & 27 & $(1,0,0)$ & $\pi$ \\
\hline 25 & 19 & $\left(0, C_{3},-1\right)$ & $2 \pi / 5$ & 58 & 28 & $(1,0,0)$ & $\pi$ \\
\hline 26 & 20 & $\left(0, C_{3},-1\right)$ & $2 \pi / 5$ & 59 & 29 & $(1,0,0)$ & $\pi$ \\
\hline 27 & 21 & $\left(0, C_{3},-1\right)$ & $2 \pi / 5$ & 60 & 30 & $(1,0,0)$ & $\pi$ \\
\hline 28 & 22 & $\left(0, C_{3},-1\right)$ & $2 \pi / 5$ & & & & \\
\hline 29 & 23 & $\left(0, C_{3},-1\right)$ & $2 \pi / 5$ & & & & \\
\hline 30 & 24 & $\left(0, C_{3},-1\right)$ & $2 \pi / 5$ & & & & \\
\hline
\end{tabular}

\section{References}

[1] T. J. R. Hughes, J. A. Cottrell, and Y. Bazilevs, "Isogeometric analysis: CAD, finite elements, NURBS, exact geometry and mesh refinement," Computer Methods in Applied Mechanics and Engineering, vol. 194, no. 39, pp. 41354195, 2005.

[2] V. P. Nguyen, C. Anitescu, S. P. A. Bordas, and T. Rabczuk, "Isogeometric 
analysis: An overview and computer implementation aspects," Mathematics and Computers in Simulation, vol. 117, pp. 89-116, 2015.

[3] J. A. Cottrell, T. J. Hughes, and Y. Bazilevs, Isogeometric Analysis: Toward Integration of CAD and FEA. Chichester: Wiley, 2009.

[4] L. Piegl and W. Tiller, The NURBS Book. Berlin, Heidelberg: SpringerVerlag, 2nd ed., 1997.

[5] J. E. Cobb, "Tiling the sphere with rational Bézier patches," tech. rep., University of Utah, 1988.

[6] Y. Bazilevs, V. M. Calo, J. A. Cottrell, J. A. Evans, T. J. R. Hughes, S. Lipton, M. A. Scott, and T. W. Sederberg, "Isogeometric analysis using T-splines," Computer Methods in Applied Mechanics and Engineering, vol. 199, pp. 229263, 2010.

[7] M. A. Scott, X. Li, T. W. Sederberg, and T. J. R. Hughes, "Local refinement of analysis-suitable T-splines," Computer Methods in Applied Mechanics and Engineering, vol. 213, pp. 206-222, 2012.

[8] D. Burkhart, B. Hamann, and G. Umlauf, "Iso-geometric Finite Element Analysis Based on Catmull-Clark Subdivision Solids," Computer Graphics Forum, vol. 29, pp. 1575-1584, sep 2010.

[9] X. Wei, Y. Zhang, T. Hughes, and M. Scott, "Truncated hierarchical CatmullClark subdivision with local refinement," Computer Methods in Applied Mechanics and Engineering, vol. 291, pp. 1-20, 2015.

[10] X. Zhu, P. Hu, and Z.-D. Ma, "B++ splines with applications to isogeometric analysis," Computer Methods in Applied Mechanics and Engineering, vol. 311, pp. 503-536, 2016.

[11] G. Xu, E. Atroshchenko, and S. P. A. Bordas, "Geometry-independent field approximation for spline-based finite element methods," in Proceedings of the 11th World Congress in Computational Mechanics, 2014.

[12] B. Marussig, J. Zechner, G. Beer, and T.-P. Fries, "Fast isogeometric boundary element method based on independent field approximation," Computer Methods in Applied Mechanics and Engineering, vol. 284, pp. 458-488, 2015.

[13] L. Coox, F. Greco, O. Atak, D. Vandepitte, and W. Desmet, "A robust patch coupling method for NURBS-based isogeometric analysis of non-conforming multipatch surfaces," Computer Methods in Applied Mechanics and Engineering, vol. 316, pp. 235-260, 2017. 
[14] Z. Zou, M. A. Scott, M. J. Borden, D. C. Thomas, W. Dornisch, and E. Brivadis, "Isogeometric Bézier dual mortaring: Refineable higherorder spline dual bases and weakly continuous geometry," arXiv preprint arXiv:1711.01009, 2017.

[15] I. Babuška, "The finite element method with Lagrangian multipliers," Numerische Mathematik, vol. 20, no. 3, pp. 179-192, 1973.

[16] I. Babuška, "The Finite Element Method with Penalty," Mathematics of Computation, vol. 27, no. 122, pp. 221-228, 1973.

[17] J. Nitsche, "Über ein Variationsprinzip zur Lösung von Dirichlet-Problemen bei Verwendung von Teilräumen, die keinen Randbedingungen unterworfen sind," Abhandlungen aus dem Mathematischen Seminar der Universität Hamburg, vol. 36, no. 1, pp. 9-15, 1971.

[18] V. P. Nguyen, P. Kerfriden, M. Brino, S. P. A. Bordas, and E. Bonisoli, "Nitsche's method for two and three dimensional NURBS patch coupling," Computational Mechanics, vol. 53, no. 6, pp. 1163-1182, 2014.

[19] C. Bernardi, Y. Maday, and T. Patera, "Domain Decomposition by the Mortar Element Method," in Asymptotic and Numerical Methods for Partial Differential Equations with Critical Parameters, vol. 384, ch. 6, pp. 269-286, Kluwer Academic Publishers, 1993.

[20] F. Ben Belgacem, "The Mortar finite element method with Lagrange multipliers," Journal of Numerical Mathematics, vol. 84, pp. 173-197, 1999.

[21] W. Dornisch, G. Vitucci, and S. Klinkel, "The weak substitution method an application of the mortar method for patch coupling in NURBS-based isogeometric analysis," International Journal for Numerical Methods in Engineering, vol. 103, pp. 205-234, 2015.

[22] A. Goyal and B. Simeon, "On penalty-free formulations for multipatch isogeometric Kirchhoff-Love shells," Mathematics and Computers in Simulation, vol. 136, pp. 78-103, 2017.

[23] J. Kiendl, Y. Bazilevs, M.-C. Hsu, R. Wüchner, and K.-U. Bletzinger, "The bending strip method for isogeometric analysis of Kirchhoff-Love shell structures comprised of multiple patches," Computer Methods in Applied Mechanics and Engineering, vol. 199, no. 37, pp. 2403-2416, 2010.

[24] Y. Guo and M. Ruess, "Nitsche's method for a coupling of isogeometric thin shells and blended shell structures," Computer Methods in Applied Mechanics and Engineering, vol. 284, pp. 881-905, 2015. 
[25] F. Maurin, L. Coox, F. Greco, E. Deckers, C. Claeys, and W. Desmet, "Bloch theorem for isogeometric analysis of periodic problems governed by high-order partial differential equations," Computer Methods in Applied Mechanics and Engineering, vol. 311, pp. 743-763, 2016.

[26] T. X. Duong, F. Roohbakhshan, and R. A. Sauer, "A new rotation-free isogeometric thin shell formulation and a corresponding continuity constraint for patch boundaries," Computer Methods in Applied Mechanics and Engineering, vol. 316, pp. 43-83, 2017.

[27] L. Coox, F. Maurin, F. Greco, E. Deckers, D. Vandepitte, and W. Desmet, "A flexible approach for coupling NURBS patches in rotation-free isogeometric analysis of Kirchhoff-Love shells," Computer Methods in Applied Mechanics and Engineering, vol. 325, pp. 505-531, 2017.

[28] R. Hartshorne, Algebraic Geometry. New York: Springer-Verlag, 1977.

[29] R. Dietz, J. Hoschek, and B. Jüttler, "An algebraic approach to curves and surfaces on the sphere and on other quadrics," Computer Aided Geometric Design, vol. 10, no. 3-4, pp. 211-229, 1993.

[30] R. Dietz, J. Hoschek, and B. Jüttler, "Rational patches on quadric surfaces," Computer-Aided Design, vol. 27, no. 1, pp. 27-40, 1995.

[31] "Octave NURBS Toolbox." https://octave.sourceforge.io/nurbs/, 2017.

[32] P. R. Cromwell, Polyhedra. Cambridge University Press, 1999.

[33] Y. Sakano and Y. Akama, "Anisohedral spherical triangles and classification of spherical tilings by congruent kites, darts and rhombi," Hiroshima Math. J., vol. 45, pp. 309-339, 112015.

[34] G. Xu, B. Mourrain, R. Duvigneau, and A. Galligo, "Parameterization of computational domain in isogeometric analysis: methods and comparison," Computer Methods in Applied Mechanics and Engineering, vol. 200, no. 23, pp. 2021-2031, 2011.

[35] S. Lipton, J. A. Evans, Y. Bazilevs, T. Elguedj, and T. J. R. Hughes, "Robustness of isogeometric structural discretizations under severe mesh distortion," Computer Methods in Applied Mechanics and Engineering, vol. 199, no. 5, pp. 357-373, 2010. 
[36] J. A. Cottrell, A. Reali, Y. Bazilevs, and T. J. R. Hughes, "Isogeometric analysis of structural vibrations," Computer Methods in Applied Mechanics and Engineering, vol. 195, no. 41, pp. 5257-5296, 2006.

[37] Y. Bazilevs, L. Beirão da Veiga, J. A. Cottrell, T. J. R. Hughes, and G. Sangalli, "Isogeometric analysis: approximation, stability and error estimates for h-refined meshes," Mathematical Models and Methods in Applied Sciences, vol. 16, no. 07, pp. 1031-1090, 2006.

[38] E. Pilgerstorfer and B. Jüttler, "Bounding the influence of domain parameterization and knot spacing on numerical stability in Isogeometric Analysis," Computer Methods in Applied Mechanics and Engineering, vol. 268, pp. 589613, 2014.

[39] G. Xu, B. Mourrain, R. Duvigneau, and A. Galligo, "Analysis-suitable volume parameterization of multi-block computational domain in isogeometric applications," Computer-Aided Design, vol. 45, no. 2, pp. 395-404, 2013.

[40] H. Speleers and C. Manni, "Optimizing domain parameterization in isogeometric analysis based on Powell-Sabin splines," Journal of Computational and Applied Mathematics, vol. 289, pp. 68-86, 2015.

[41] E. Skudrzyk, The Foundations of Acoustics: Basic Mathematics and Basic Acoustics. Vienna: Springer-Verlag, 1971.

[42] M. C. Junger and D. Feit, Sound, structures, and their interaction. Lexington: MIT Lincoln Laboratory, 1972.

[43] R. N. Simpson, M. A. Scott, M. Taus, D. C. Thomas, and H. Lian, "Acoustic isogeometric boundary element analysis," Computer Methods in Applied Mechanics and Engineering, vol. 269, pp. 265-290, 2014.

[44] X. Peng, E. Atroshchenko, P. Kerfriden, and S. P. A. Bordas, "Isogeometric boundary element methods for three dimensional static fracture and fatigue crack growth," Computer Methods in Applied Mechanics and Engineering, vol. 316, pp. 151-185, 2017.

[45] L. Coox, O. Atak, D. Vandepitte, and W. Desmet, "An isogeometric indirect boundary element method for solving acoustic problems in open-boundary domains," Computer Methods in Applied Mechanics and Engineering, vol. 316, pp. 186-208, 2017.

[46] T. Belytschko, H. Stolarski, W. K. Liu, N. Carpenter, and J. S.-J. Ong, "Stress projection for membrane and shear locking in shell finite elements," Computer Methods in Applied Mechanics and Engineering, vol. 51, pp. 221-258, 1985. 
[47] R. H. Macneal and R. L. Harder, "A proposed standard set of problems to test finite element accuracy," Finite Elements in Analysis and Design, vol. 1, no. 1 , pp. $3-20,1985$.

[48] L. S. D. Morley and A. J. Morris, "Conflict Between Finite Elements and Shell Theory," tech. rep., Royal Aircraft Establishment, London, 1978.

[49] J. Kiendl, K.-U. Bletzinger, J. Linhard, and R. Wüchner, "Isogeometric shell analysis with Kirchhoff-Love elements," Computer Methods in Applied Mechanics and Engineering, vol. 198, no. 49, pp. 3902-3914, 2009.

[50] H. Lamb, "On the Vibrations of a Spherical Shell," Proceedings of the London Mathematical Society, vol. s1-14, pp. 50-56, nov 1882.

[51] A. E. H. Love, "The small free vibrations and deformation of a thin elastic shell," Philosophical Transactions of the Royal Society of London. A, vol. 179, pp. 491-546, 1888.

[52] A. Kalnins in Proc. Natl. Congr. Appl. Mech. 4th U.S., pp. 225-223, 1963.

[53] A. Kalnins, "Effect of Bending on Vibrations of Spherical Shells," The Journal of the Acoustical Society of America, vol. 36, pp. 74-81, jan 1964.

[54] P. M. Naghdi and A. Kalnins, "On Vibrations of Elastic Spherical Shells," Journal of Applied Mechanics, vol. 29, no. 1, p. 65, 1962.

[55] A. Silbiger, "Nonaxisymmetric Modes of Vibration of Thin Spherical Shells," The Journal of the Acoustical Society of America, vol. 34, pp. 862-862, jun 1962.

[56] H. Kraus, Thin elastic shells: an introduction to the theoretical foundations and the analysis of their static and dynamic behavior. Wiley, 1967.

[57] F. I. Niordson, "Free vibrations of thin elastic spherical shells," International Journal of Solids and Structures, vol. 20, no. 7, pp. 667-687, 1984.

[58] F. I. Niordson, "The spectrum of free vibrations of a thin elastic spherical shell," International Journal of Solids and Structures, vol. 24, no. 9, pp. 947961, 1988. 